\title{
NOTES ;ON SOME EURYHALINE GAMMARIDS (CRUSTACEA, AMPHIPODA) FROM THE WEST-COAST OF NORWAY
}

\author{
by
}

\author{
HENK G. DENNERT \\ Institute of Taxonomic Zoology, University of Amsterdam, The Netherlands
}

\begin{abstract}
Some data on the distribution in Norway and on the ecology of the euryhaline amphipods Gammarus duebeni duebeni, G. oceanicus, G. zaddachi, G. finmarchicus and Chaetogammarus marinus are presented in this paper.

Morphological differences between populations of $\boldsymbol{G}$. oceanicus are briefly discussed.
\end{abstract}

\section{INTRODUCTION}

Relatively little is known about distribution and ecology of members of the family Gammaridae in Norway. Records concerning the biology and distribution of the genera Gammarus, Chaetogammarus, and Eulimnogammarus are found in the papers of Oldevig (1933 \& 1959), Stephensen (1935-1942, with a synopsis of the then known literature), Dahl (1938, 1946 \& 1952), Sexton \& Spooner (1940), Sexton (1942), Schellenberg (1942), Segerstråle (1947, 1948 \& 1959), Spooner (1951), Steen (1951), J. Økland (1959), K.A. Økland (1965, 1969 a \& b, 1970), Brattegard (1966), Ofstad \& Solem (1966), Stock \& Kant (1966), Stock (1967), Solem (1969) and Vader (1969, 1971, 1972 a \& b).

During a sampling trip in July 1971 along the Atlantic coast of Norway an attempt was made to obtain further information about some euryhaline species of the genera mentioned above. A survey was made in four biotopes: supralittoral rockpools, small springs in seacliffs with irregular salinity changes, small estuaries, and the intertidal zone of the fjords. For practical reasons, and because the biotopes have some common characteristics (such as a certain similarity in gammarid fauna and periodical changes in salt-content of the water) the small springs in seacliffs and small estuaries were considered together and the samples from these biotopes were pooled. Especially localities where Gammarus duebeni duebeni Lilljeborg.
1852, Gammarus oceanicus Segerstråle, 1947, Gammarus zaddachi Sexton, 1912, Gammarus finmarchicus Dahl, 1938, and Chaetogammarus marinus (Leach, 1815) were found, will be used in comparing the four biotopes. In the investigated biotopes these five species were most abundant. Other euryhaline gammarids, as Gammarus salinus Spooner, 1947, Gammarus setosus Dementieva, 1931, Chaetogammarus stoerensis (Reid, 1938) and Eulimnogammarus obtusatus (Dahl, 1938), together with a number of other species belonging to the Gammaridae and Talitridae, were found at several of the visited localities.

An extensive study of the limnic waterbodies and rivers in Norway was not made, since Økland (1969 a \& b) reported in detail about this subject. In all inland waters, Gammarus lacustris Sars, 1863, is the main representative.

At some inland localities Gammarus duebeni is found, but in a following paper it will be shown that these localities always have a mixohaline nature, and the subspecies found there is the brackishwater form, G. duebeni duebeni. For further information regarding G. duebeni I refer to Pinkster et al. (1970) and Sutcliffe (1972).

In studying the samples it became apparent, that some taxonomic characters used in distinguishing G. oceanicus from other Gammarus species, varied in the populations examined. In a separate chapter (III) these differences will be discussed.

\section{METHODS}

At all 177 localities visited temperature and specific conductivity of the water was determined. From a water-sample both $\mathrm{Cl}^{-}$and $\mathrm{Ca}^{++}$contents were determined in Amsterdam.

Collected specimens have been deposited in the collection of the Zoölogisch Museum of the University of Amsterdam. 


\section{DIFFERENCES IN MORPHOLOGY BE- TWEEN G. OCEANICUS POPULATIONS}

As pointed out in the introduction, several populations of $G$. oceanicus show characteristics deviating from the typical distinguishing characters as introduced by Segerstråle (1947) and used by Spooner (1951) and Kinne (1954). In these populations, which include ovigerous females, the males differ from the typical $G$. oceanicus in the following characters:

- The A2 flagellum is devoid of calceoli.

- The third segment of the mandible palp is ventrally armed with a row of spinules, which diminish regularly in size in proximal direction.

- Segment 1 of A1 bears on its ventral surface one thin tuft of setae and an apical tuft of setae; segment 2 bears three or four tufts of setae (including the apical tuft). The setae of the tufts on both the first and second seg- ment of A1 are often somewhat shorter than the setae of the corresponding tufts in typical G.oceanicus males.

Typical G. oceanicus males show the following features:

- An A2 flagellum with calceoli.

- The third segment of the mandible palp is ventrally armed with a row of spinules which are mutually of a size.

- Segment 1 of A1 carries on its ventral surface two (rarely a small third) tufts of setae and an apical tuft; segment 2 has four or five tufts of setae (including the apical tuft).

Confusion thus might be caused between the atypical $G$. oceanicus and some representatives of the $G$. locusta-group (e.g., G. inaequicauda Stock, 1966) but can be prevented by observing certain other typical $G$. oceanicus characters:

The median palmar spine of the propodus of the
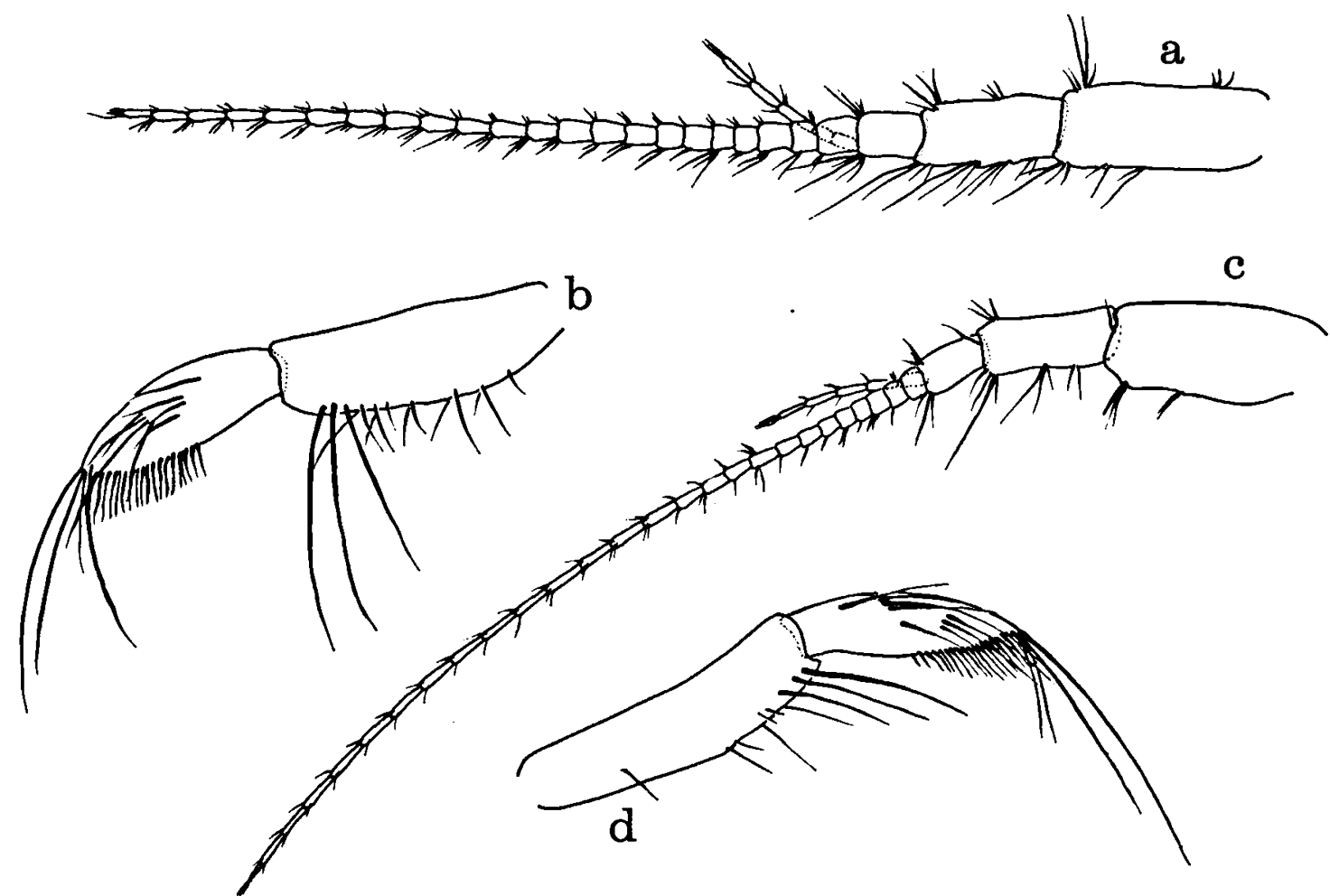

$2 \mathrm{~mm}$ scale 1

\section{$1 \mathrm{~mm}$ scale 2}

Fig. 1. G. oceanicus: $\hat{o}$ of the typical form, from fjord at Mandal (body length $16.5 \mathrm{~mm}$ ) and $\hat{\delta}$ of the atypical form, from Straumfjorden at Straumfjordnes (body length $16.3 \mathrm{~mm}$ ).

a, first antenna $\hat{\delta}$ typical form (scale 1); b, mandible palp $\hat{\delta}$ typical form (scale 2); c, first antenna $\hat{\delta}$ atypical form (scale 1); d, mandible palp ô atypical form (scale 2). 
Table I

Percentage of joint occurrence of Gammarus, Chaetogammarus, and Eulimnogammarus species in 3 investigated biotopes.
G. duebeni
G. oceanicus
all samples
G. oceanicus
atypical form
G. zaddachi
G. finmarchicus Ch. marinus

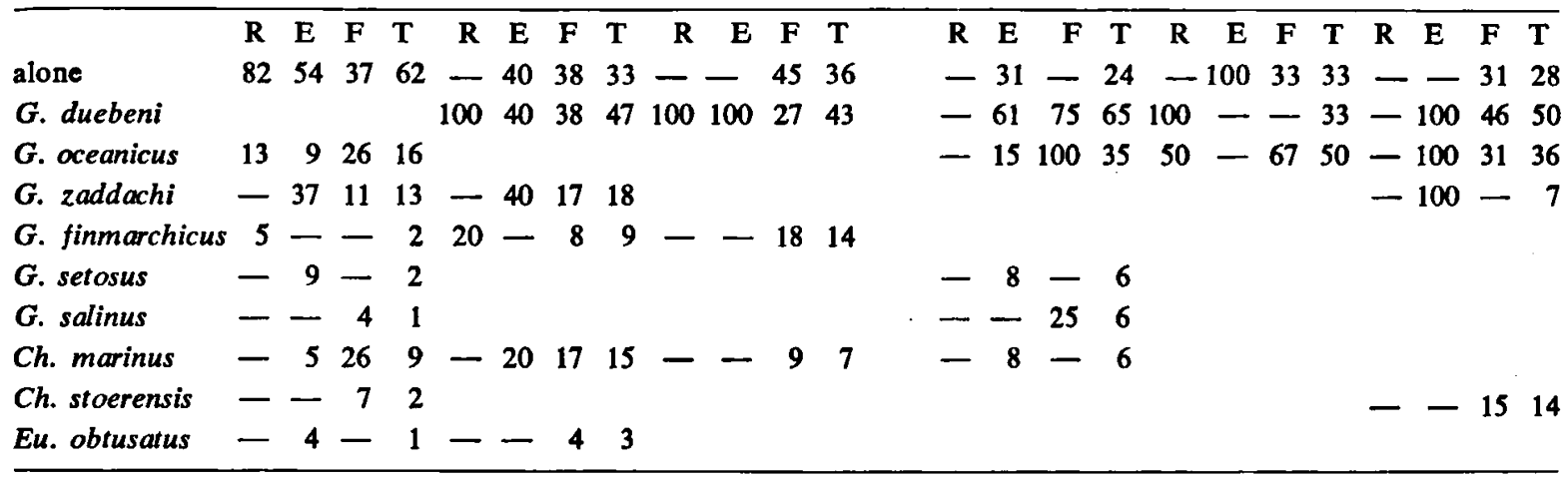

total number

of the samples

percentage of the samples in each biotope for each species

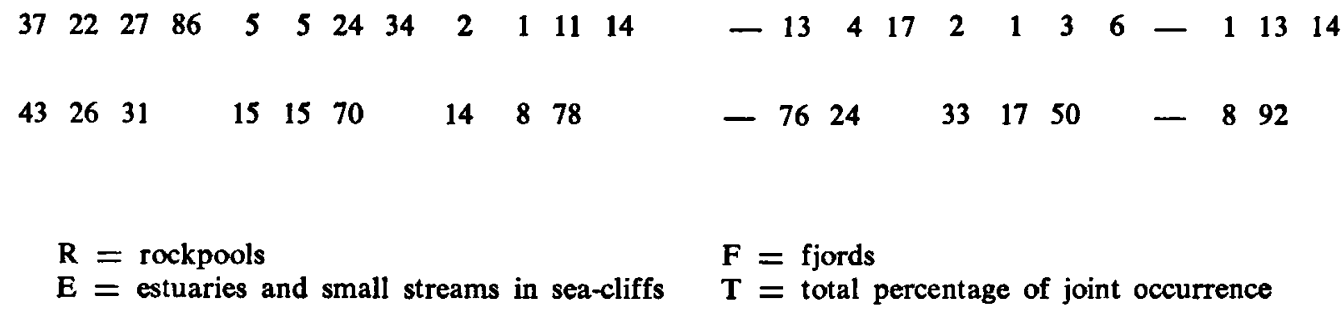

second leg is pointed and tapering (not "flaskshaped"), the propodus of the second leg is roundish, the exopod of the third uropod has many plumose setae, and the lateral lobes of the head are of the Lagunogammarus-type (see Sket, 1971) which is for instance found in $G$. zaddachi, $G$. oceanicus and $G$. salinus. In fig. 1 the differences between the typical and atypical form are illustrated. The localities where the atypical form was found are indicated on the map in fig. 3.

In the southern part of Norway only specimens of the typical form were found, more to the north mixed populations and populations with the atypical form only. Neither in the biotopes inhabited by the two forms, nor in the way they live associated with other Gammarus species any difference was found between the two forms (cf. table I, chapter IV). However, it seems plausible to suppose that the variability mentioned above may be influenced by temperature and possibly by other factors. These factors may have a considerable effect on the development of the animals in a way we do not yet quite understand and may cause the morphological variability described.

\section{DISTRIBUTION OF THE INVESTIGATED SPECIES ALONG THE ATLANTIC COAST OF NORWAY.}

The maps (figs. 2 and 3 ) indicate the stations at which the gammarids have been collected in Norway. Stations at which no gammarids were found are not indicated to avoid confusion. The appendix contains a list of all the localities with gammarids and some ecological data of each locality. This list includes a great number of new records of the species concerned.

In table I the joint occurence of the species as well as the frequency in each of the three biotopes investigated are summarized.

It shows, that $G$. duebeni duebeni inhabits mainly the supralittoral rockpools. Only in pseudo-supralittoral rockpools, situated in the lowest part of the black zone, at the boundary of the eulittoral and supralittoral, the species coexists with G. oceanicus and G. finmarchicus. In small estuaries and at places where springs in seacliffs reach the sea, the species lives associated with $G$. zaddachi. The substratum is mostly sand or mud with 


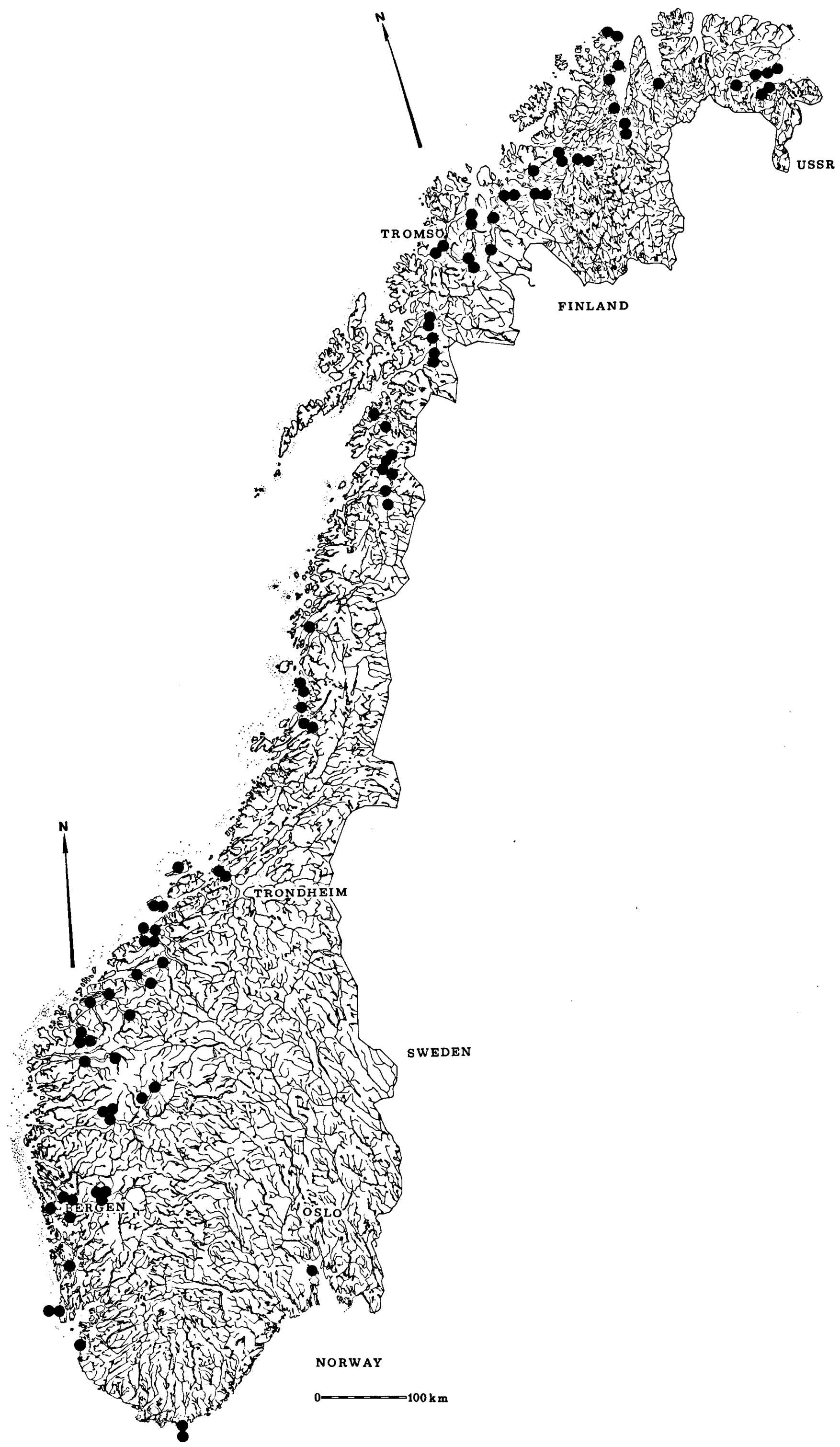

Fig. 2. Positive stations with G. duebeni duebeni in Norway. 


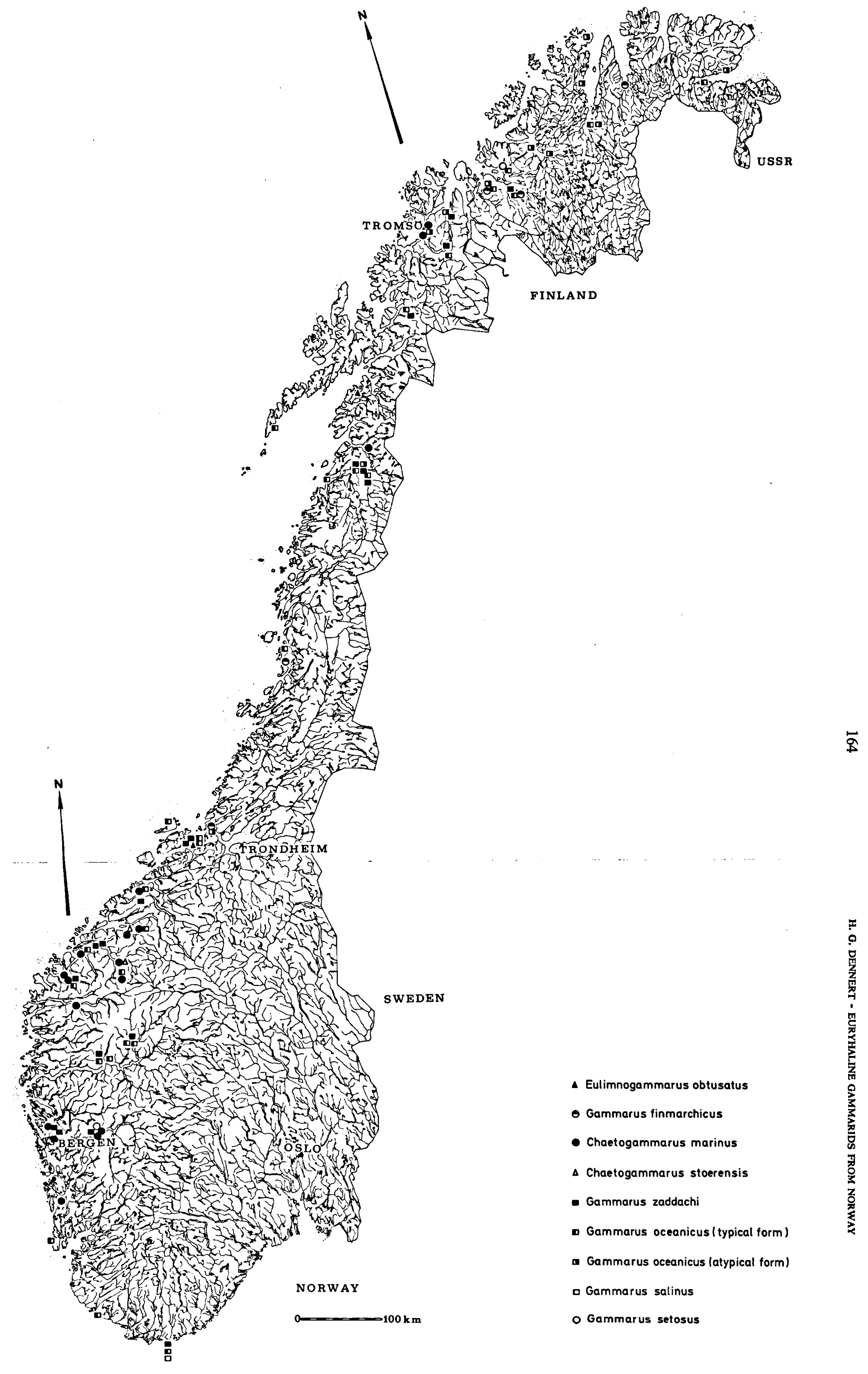

Fig. 3. Positive stations with gammarids, except G. duebeni duebeni, in Norway. 
scattered boulders. In fjords, $\boldsymbol{G}$. duebeni is practically limited to the upper algae zones with Pelvetia and Ascophyllum and is sometimes found intertidally on gravel beaches. This species lives here mainly in association with $G$. oceanicus on algae and with $C h$. marinus under boulders.

G. oceanicus occupies chiefly the eulittoral zone of the fjords, where the species is found in fairly shallow water among stones, on Fucus and other algae, on a bottom of sand or pebbles. In this habitat $G$. oceanicus occurs together with $G$. duebeni, but also with $G$. zaddachi and $C h$. marinus. The substratum in running waters, e.g. mouths of small rivers, is sand or sandy mud with an Enteromorpha vegetation and here the species coexists with G. duebeni and G. zaddachi. In pseudo-supralittoral rockpools $G$. oceanicus lives associated with $G$. duebeni and $G$. finmarchicus.

$G$. zaddachi lives mostly in running waters, and never in rockpools. In estuaries the species is associated mainly with $G$. duebeni, in fjords adjacent to estuaries it is associated with $G$. duebeni and $G$. oceanicus. In the estuaries the substratum consists of sand or mud, in the fjords, in the highest littoral zone where the species occurs, the substra- tum is sand or gravel.

Ch. marinus was never found in rockpools, and was encountered only once in an estuary. In the Fucus zone of the fjords (the main biotope of Ch. marinus), the substratum on which the animals were found consists of rock or boulders. Here G. duebeni is a common companion of $C h$. marinus.

From the few samples available containing G. finmarchicus, a certain preference of this species for the littoral zone of the fjords and pseudosupralittoral rockpools can be discerned. The substratum on which the animal was found was rock or sand, sometimes covered with algae from the Fucus zone. The species occurs together with $G$. duebeni and $G$. oceanicus.

In the figs. $4 \mathrm{~A}$ and $4 \mathrm{~B}$ the localities at which the gammarids were caught are classified with regard to the type of biotope and the contents of $\mathrm{Cl}^{-}$and $\mathrm{Ca}^{++}$-ions of the water. Table II summarizes the minimum and maximum values of the $\mathrm{Cl}^{-}$and $\mathrm{Ca}^{++}$ contents, the specific conductivity and temperature (at daytime) of the water of the biotopes from which G. duebeni duebeni, G. oceanicus, G. zaddachi, G. finmarchicus and Ch. marinus were collected.

Table II

Minimum and maximum values of $\mathrm{Cl}^{-}$and $\mathrm{Ca}^{++}$contents, specific conductivity and temperature of the water of the biotopes inhabited by the five species investigated in Norway.

\begin{tabular}{|c|c|c|c|c|c|c|c|c|c|}
\hline & & \multicolumn{2}{|c|}{$\mathrm{Cl}-$ in $\mathrm{mg} / \mathrm{l}$} & \multicolumn{2}{|c|}{$\mathrm{Ca}^{++}$in $\mathrm{mg} / \mathrm{l}$} & \multicolumn{2}{|c|}{$\begin{array}{l}\text { Spec. conductivity } \\
\text { in } \mu S\end{array}$} & \multicolumn{2}{|c|}{ temperature in ${ }^{\circ} \mathrm{C}$} \\
\hline & & $\min$. & $\max$ & $\min$. & $\max$ & $\min$. & $\max$ & min. & $\max$ \\
\hline \multirow[t]{3}{*}{ G. duebeni } & $\mathbf{R}$ & 15 & 20000 & $<5$ & 376 & 170 & 36230 & 8.6 & 28.6 \\
\hline & $\mathbf{E}$ & 4 & 17000 & $<5$ & 228 & 45 & 34500 & 7.4 & 18.4 \\
\hline & $\mathbf{F}$ & 18 & 17900 & $<5$ & 376 & 85 & 33300 & 9.8 & 18.6 \\
\hline \multirow[t]{3}{*}{ G. oceanicus } & $\mathbf{R}$ & 4600 & 16100 & 84 & 276 & 11060 & 36040 & 9.0 & 14.1 \\
\hline & $\mathbf{E}$ & 5 & 12700 & $<5$ & 302 & 45 & 23970 & 8.3 & 21.0 \\
\hline & $\mathbf{F}$ & 18 & 17900 & 14 & 385 & 85 & 35250 & 9.8 & 18.2 \\
\hline \multirow[t]{3}{*}{ G. zaddachi } & $\mathbf{R}$ & - & - & - & 一 & - & - & - & - \\
\hline & $\mathbf{E}$ & 5 & 69 & $<5$ & 17 & 45 & 395 & 8.3 & 17.8 \\
\hline & $\mathbf{F}$ & 18 & 6100 & 14 & 118 & 85 & 15820 & 13.4 & 17.0 \\
\hline \multirow[t]{3}{*}{ G. finmarchicus } & $\mathbf{R}$ & 11600 & 12100 & 257 & 261 & 24890 & 25310 & 12.0 & 14.1 \\
\hline & $\mathbf{E}$ & \multicolumn{2}{|c|}{$2450^{*}$} & \multicolumn{2}{|c|}{$63^{*}$} & \multicolumn{2}{|c|}{$7200^{*}$} & \multicolumn{2}{|c|}{$10.4^{*}$} \\
\hline & $\mathbf{F}$ & 10900 & 17000 & 204 & 357 & 23960 & 33520 & 10.8 & 12.4 \\
\hline \multirow[t]{3}{*}{ Ch. marinus } & $\mathbf{R}$ & - & - & - & - & - & - & - & 一 \\
\hline & $\mathbf{E}$ & \multicolumn{2}{|c|}{$5^{*}$} & \multicolumn{2}{|c|}{$<5^{*}$} & \multicolumn{2}{|c|}{$45^{*}$} & \multicolumn{2}{|c|}{$13.2^{*}$} \\
\hline & $\mathbf{F}$ & 2000 & 17500 & 38 & 367 & 5850 & 33300 & 10.4 & 22.4 \\
\hline $\begin{array}{l}*=\text { only one st } \\
R=\text { rockpools }\end{array}$ & & & & \multicolumn{6}{|c|}{$\begin{array}{l}\mathbf{E}=\text { estuaries and small streams in sea-cliffs } \\
\mathbf{F}=\text { fjords }\end{array}$} \\
\hline
\end{tabular}




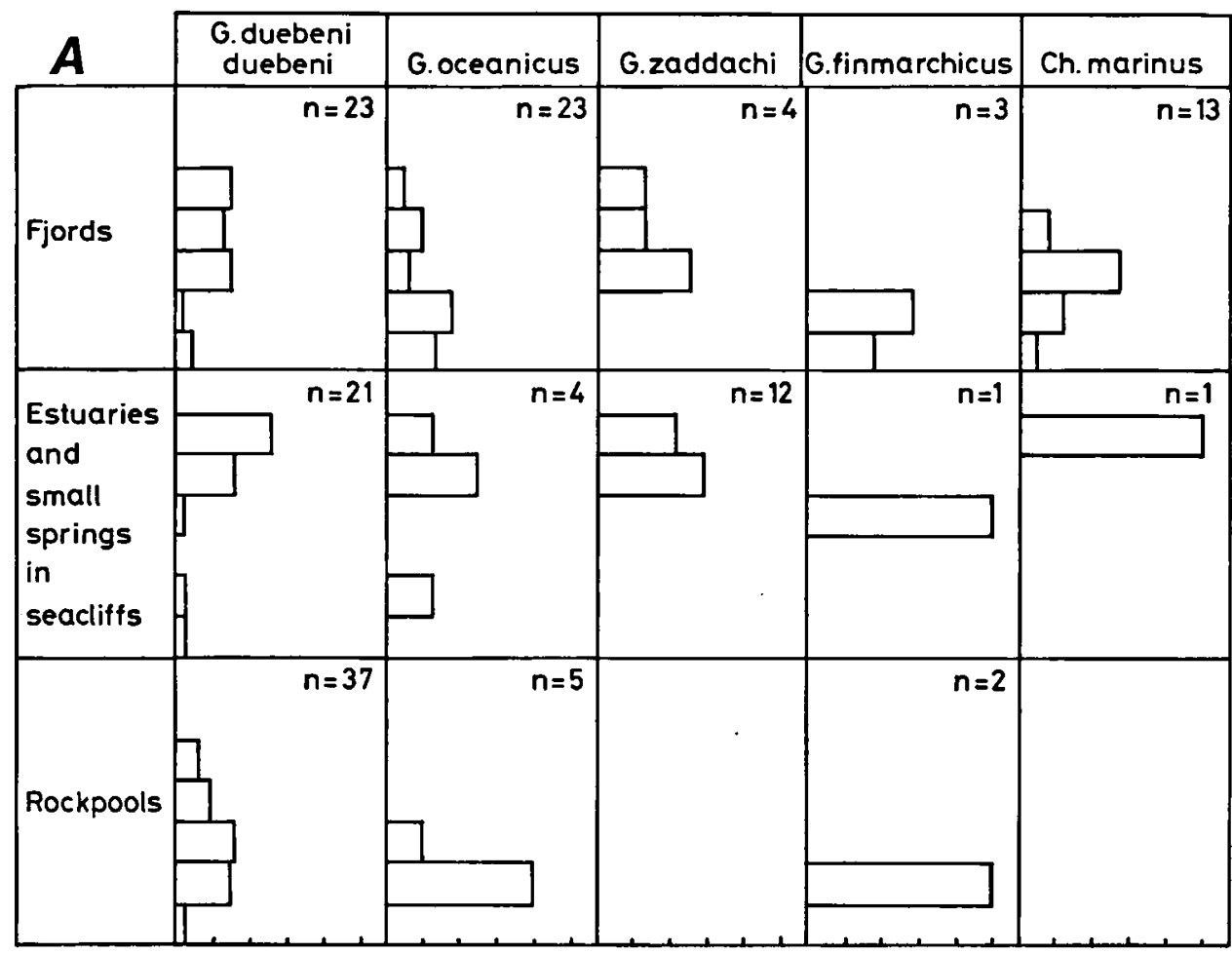

$$
\begin{aligned}
& \mathrm{mg} / 1 \mathrm{Cl}^{-} \\
0 & \rightarrow 10 \\
11 & \rightarrow 300 \\
301 & \rightarrow 3000 \\
3001 & \rightarrow 10.000 \\
10.001 & \rightarrow 16.500 \\
16.501 & \rightarrow 22.000 \\
0 & \rightarrow 10 \\
11 & \rightarrow 300 \\
301 & \rightarrow 3000 \\
3001 & \rightarrow 10.000 \\
10.001 & \rightarrow 16.500 \\
16.501 & \rightarrow 22.000 \\
0 & \rightarrow 10 \\
11 & \rightarrow 300 \\
301 & \rightarrow 3000 \\
3001 & \rightarrow 10.000 \\
10.001 & \rightarrow 16.500 \\
16.501 & \rightarrow 22.000
\end{aligned}
$$

\section{B}

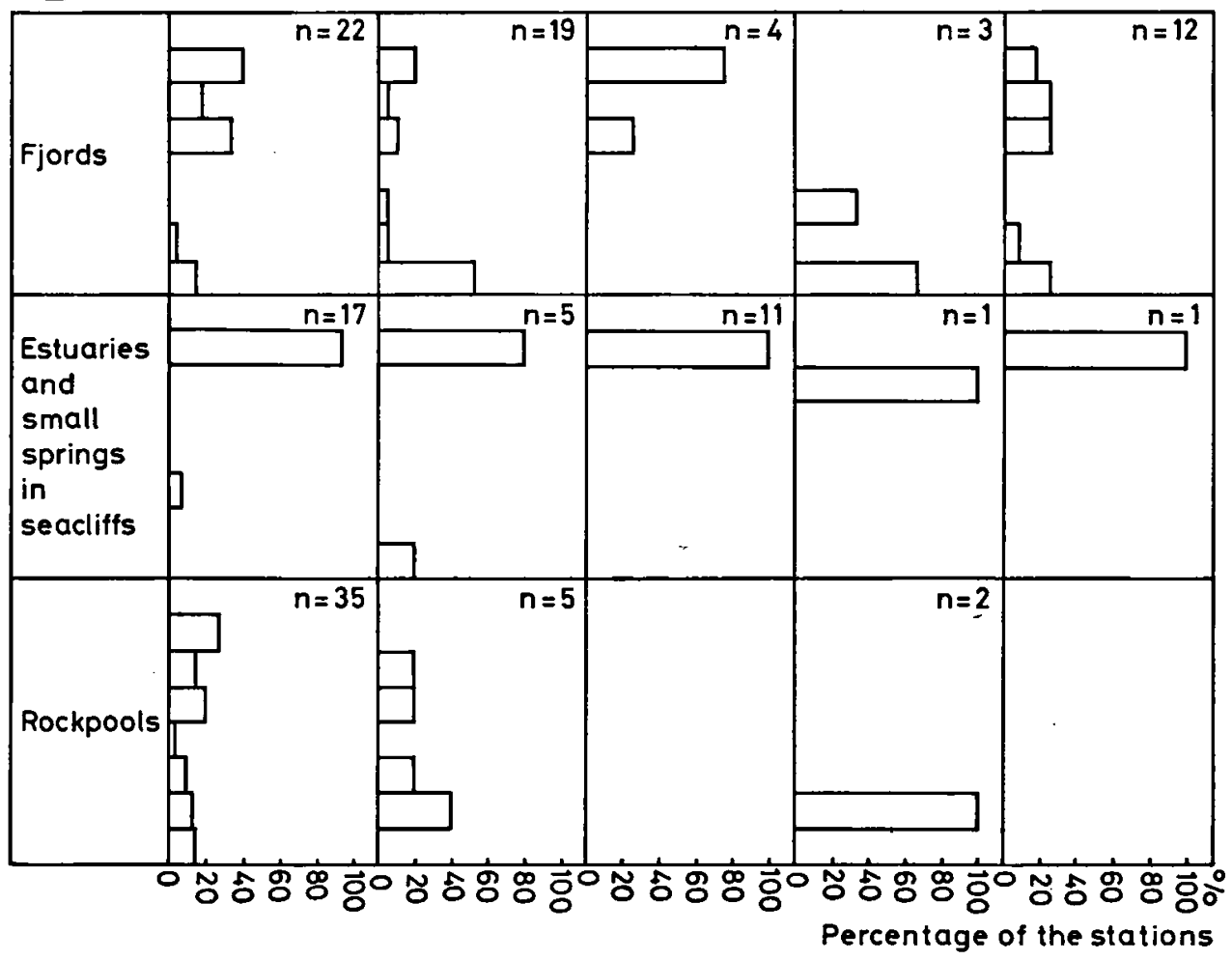

$$
\begin{aligned}
\mathrm{mg} & / 1 \mathrm{Ca}++ \\
0 & \rightarrow 50 \\
51 & \rightarrow 100 \\
101 & \rightarrow 150 \\
151 & \rightarrow 200 \\
201 & \rightarrow 250 \\
251 & \rightarrow 300 \\
301 & \rightarrow 400 \\
0 & \rightarrow 50 \\
51 & \rightarrow 100 \\
101 & \rightarrow 150 \\
151 & \rightarrow 200 \\
201 & \rightarrow 250 \\
251 & \rightarrow 300 \\
301 & \rightarrow 400 \\
0 & \rightarrow 50 \\
51 & \rightarrow 100 \\
101 & \rightarrow 150 \\
151 & \rightarrow 200 \\
201 & \rightarrow 250 \\
251 & \rightarrow 300 \\
301 & \rightarrow 400
\end{aligned}
$$

Fig. 4A. Localities with the species investigated classified with regard to the $\mathrm{Cl}^{-}$content of the water and the type of biotope.

Fig. 4B. Localities with the five species investigated classified with regard to the $\mathrm{Ca}^{++}$content of the water and the type of biotope. ( $n=$ number of stations). 


\section{V.DISCUSSION}

The facts and figures presented in chapter IV about physical and chemical environment characters, and the substratum on which the investigated species occur, agree fairly well with the scattered facts known from other publications, which, however, are mainly concerned with localities outside Norway, while in this paper a systematic investigation is made on the variability of these environmental parameters in relation to the investigated species in Norway.

Habitat selection of the species mentioned in this paper is discussed earlier in a more general way by Steen (1951), Brattegard (1966) and Meadows \& Campbell (1972).

Specifically it can be said, that the observations about habitat (including $\mathrm{Cl}^{-}$content of the water) of $G$. duebeni duebeni are in agreement with the findings of Kinne (1953a, 1954), Hynes (1954), Sutcliffe $(1967,1971)$ and Pinkster et al. (1970).

Up to now G. oceanicus had never been found at very low salinities. According to Serventy (1935), Segerstråle (1947, 1959), and Steele \& Steele (1972) this species is limited to waters with a salinity of more than $2.5 \%$, which corresponds with about $1500 \mathrm{mg} / 1 \mathrm{Cl}^{-}$. According to Steen (1951) the species is found at salinities down to $1 \%$ (corresponding with a $\mathrm{Cl}^{-}$content of about $600 \mathrm{mg} / \mathrm{l}$ ). In seven of the present records of $G$. oceanicus in Norway the chlorinity of the water was less then $600 \mathrm{mg} / 1$ (see appendix). A much lower minimum salinity must therefore be assumed for this species. This can be explained by the low temperature in northern areas, because Kinne (1952, 1953b) has provided experimental evidence that low temperatures favour survival of Gammarus at low salinities.

The data presented concerning G. zaddachi are in complete agreement with those known from the literature (Sexton 1912, Segestråle 1947, Kinne 1954, Stock, Nijssen \& Kant 1966, Dennert et al. 1969).

G. finmarchicus is found in Norway exclusively in mixohaline waters of rockpools and in the tidal belt of fjords. Neither on the $\mathrm{Cl}^{-}$and $\mathrm{Ca}^{++}$contents of the water, nor about the other environmental factors discussed here, exact observations are known. Dahl (1938, 1946), Sexton \& Spooner (1940) and Brattegard (1966) mention the lower half of the littoral zone as the typical biotope for this species.

In one case Ch. marinus is found in an estuary with an extreme low salinity at low tide. Jones (1948) notes the occurrence of Ch. marinus in estuaries in Britain, but fails to give minimum values of the salinity at ebbtide. At hightide the chlorinity of the water is $5500 \mathrm{mg} / 1 \mathrm{Cl}^{-}$. Robelus (1970, unpublished) gives as minimum value at which he found Ch. marinus in small streams in the cliffs of the French Channel coast, a $\mathrm{Cl}^{-}$content of 74 $\mathrm{mg} / 1$. In the intertidal belt in the Netherlands, Den Hartog (1964) and Vlasblom \& Bolier (1971) found populations at a minimum chlorinity of $7000 \mathrm{mg} / 1$ $\mathrm{Cl}^{-}$.

From the data presented it can be concluded, that $G$. oceanicus and $C h$. marinus occur at a wider salinity range than was known from the literature. No distinction can be made between the typical and atypical form of $G$. oceanicus with regard to the environmental factors discussed above. Joint occurrence of two or more of the investigated species is possible in all biotopes studied (see table I). When the conditions become suboptimal for some of the species, the numbers of associated species drop. Finally, in the most extreme biotope, the supralittoral rockpools, in $82 \%$ of the positive stations the only species found is G.duebeni duebeni.

\section{ACKNOWLEDGEMENTS}

The author is indebted to Prof. Dr. J. H. Stock for his advice in taxonomic problems, and to Mr. J. Zaagman for his assistence in making the graphs. Furthermore I owe thanks to Drs. A. L. Dennert for her help during the fieldwork and the chemical determinations, to Mr. $R$. Robelus for the samples he put at my disposal, and to the Netherlands' Organization for Pure Scientific Research (ZWO) for a grant, that made the fieldwork possible.

\section{APPENDIX}

\section{LIST OF LOCALITIES WHERE GAMMARIDS WERE} COLLECTED

G. duebeni was found at the stations: $1-5,8-14,17-19$, $21-24,26-30,32,34-43,47,48,50,54-57,59-62,65-70$, $72-74,76-79,81,83-90,92-94,97,98,100-106,108-112$, 115-117.

G. oceanicus was found at the stations: $2,6,7,18,22$, $25,27,31,33,37,44,47,50,51,53,59,63,64,75,77$, $80,84,87,89,91,93,95,97,99,101,104,107,109$, 113, 114, 118.

G. zaddachi was found at the stations: $2,10,12,18,22$, $27,30,45,46,52,53,61,64,76,82,86,92,96$.

G. finmarchicus was found at the stations: $50,58,71$, 91, 93, 110.

G. setosus was found at the stations 12 and 94 .

G. salinus was found at station 2 .

Ch. stoerensis was found at the stations 39 and 40.

Ch. marinus was found at the stations: 13-16, 20, 26, 27, $31,33,37,39,40,44,84$.

Eul. obtusatus was found at the stations 51 and 94. 


\section{LIST OF STATIONS}

Prov. Vestfold:

1 - Ditch near Borrevann, pos.: $59^{\circ} 25^{\prime} 30^{\prime \prime} \mathrm{N}, 10^{\circ} 25^{\prime}$ $50^{\prime \prime}$, 29-VIII-1958, brackish water (to the taste).

Prov. Vest-Agder:

2 - Fjord at camping Mandal, Mandal, pos.: 58 ${ }^{\circ} 1^{\prime}$ $\mathrm{N}, 7^{\circ} 26^{\prime} 30^{\prime \prime} \mathrm{E}, 1-\mathrm{VII}-1971$, temp. $16.2^{\circ} \mathrm{C}, \mathrm{Cl}-6100$

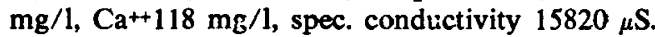

3 - Rockpools near fjord at camping Mandal, pos.: $58^{\circ} 01^{\prime} \mathrm{N}, 7^{\circ} 26^{\prime} 30^{\prime \prime} \mathrm{E}, 1-\mathrm{VII}-1971$, temp. $24.5^{\circ} \mathrm{C}$, $\mathrm{Cl}-105 \mathrm{mg} / \mathrm{l}$, spec. conductivity $420 \mu \mathrm{S}$.

Prov. Rogaland:

4 - Rockpool at sea at Tananger, pos.: $58^{\circ} 56^{\prime} 20^{\prime \prime} \mathrm{N}$, $5^{\circ} 34^{\prime} \mathrm{E}, 2$-VII-1971, temp. $17.0^{\circ} \mathrm{C}, \mathrm{Ca}^{++} 241 \mathrm{mg} / \mathrm{l}$, spec. conductivity $31640 \mu \mathrm{S}$.

5 - Rockpool at Syrafjorden, Ferringstadhamn, pos.: $59^{\circ} 13^{\prime} 40^{\prime \prime} \mathrm{N}, 5^{\circ} 10^{\prime} \mathrm{E}, 2-\mathrm{VII}-1971$, temp. $16.0^{\circ} \mathrm{C}, \mathrm{Cl}-$ $8100 \mathrm{mg} / \mathrm{l}, \mathrm{Ca}++118 \mathrm{mg} / \mathrm{l}$, spec. conductivity $18460 \mu \mathrm{S}$.

6 - Söndregåpet, Hestnes, pos.: $58^{\circ} 26^{\prime} \mathrm{N}, 6^{\circ} 01^{\prime} \mathrm{E}$, 1-VII-1971, temp. $13.8^{\circ} \mathrm{C}, \mathrm{Cl}-10500 \mathrm{mg} / \mathrm{l}$, spec. conductivity $21700 \mu \mathrm{S}$.

7 - Mouth river at Ferkingstadhamn, pos.: $59^{\circ} 13^{\prime} 40^{\prime \prime}$ $\mathrm{N}, 5^{\circ} 10^{\prime} 30^{\prime \prime} \mathrm{E}, 2-\mathrm{VII}-1971$, temp. $21.0^{\circ} \mathrm{C}, \mathrm{Cl}^{-167}$ $\mathrm{mg} / \mathrm{l}, \mathrm{Ca}^{++14} \mathrm{mg} / \mathrm{l}$.

Prov. Hordaland:

8 - Rockpool at Bömlafjorden, Valevåg, pos.: 59 $42^{\prime} \mathrm{N}, 5^{\circ} 29^{\prime} \mathrm{E}, 2$-VII-1971, temp. $22.6^{\circ} \mathrm{C}, \mathrm{Cl}-20000$

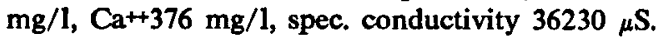

9 - Mouth cliffstream in side-branch of Kobbaleiafjord, Kolltveit, pos.: $60^{\circ} 20^{\prime} 40^{\prime \prime} \mathrm{N}, 5^{\circ} 03^{\prime} \mathrm{E}, 4$-VII1971 , temp. $18.4^{\circ} \mathrm{C}, \mathrm{Cl}^{-14} \mathrm{mg} / \mathrm{l}, \mathrm{Ca}^{++}<5 \mathrm{mg} / \mathrm{l}$. spec. conductivity $70 \mu \mathrm{S}$.

10 - River under road 560, between Ytre Arna and Arna, pos.: $60^{\circ} 27^{\prime} \mathrm{N}, 5^{\circ} 27^{\prime} 30^{\prime \prime} \mathrm{E}, 5$-VII-1971, temp. $14.8^{\circ} \mathrm{C}, \mathrm{Cl}^{-69} \mathrm{mg} / 1$, spec. conductivity $395 \mu \mathrm{S}$.

11 - Sörfjord, Ytro Arna, pos.: $60^{\circ} 27^{\prime} \mathrm{N}, 5^{\circ} 27^{\prime} 30^{\prime \prime} \mathrm{E}$, 5-VII-1971, sand beach, no watersample.

12 - Mouth of river at Ytre Samlafjorden, South of Øystese, pos.: $60^{\circ} 23^{\prime} \mathrm{N}, 6^{\circ} 12^{\prime} \mathrm{E}, 5-\mathrm{VII}-1971$, temp. $11.8^{\circ} \mathrm{C}, \mathrm{Cl}-8 \mathrm{mg} / 1, \mathrm{Ca}^{++}<5 \mathrm{mg} / \mathrm{l}$, spec. conductivity $150 \mu \mathrm{S}$.

13 - Ytre Samlafjorden, $100 \mathrm{~m}$ E. of river, Øystese, pos.: $60^{\circ} 23^{\prime} \mathrm{N}, 6^{\circ} 12^{\prime} \mathrm{E}, 5-\mathrm{VII}-1971$, temp. $17.8^{\circ}$

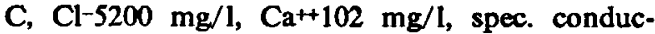
tivity $13500 \mu \mathrm{S}$.

14 - Ytre Samlafjorden, $10 \mathrm{~m} \mathrm{E}$. of river, Øystese, pos.: $60^{\circ} 23^{\prime} \mathrm{N}, 6^{\circ} 12^{\prime} \mathrm{E}, 5-\mathrm{VII}-1971$, temp. $15.9^{\circ} \mathrm{C}$, Cl-3600 mg/l, Ca++70 mg/l, spec. conductivity $8610 \mu \mathrm{S}$.

15 - Fjord S. of Salhus, pos.: $60^{\circ} 29^{\prime} 20^{\prime \prime} \mathrm{N}, 5^{\circ} 10^{\prime} 15^{\prime \prime}$

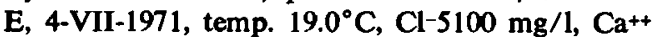
$86 \mathrm{mg} / \mathrm{l}$, spec. conductivity $11230 \mu \mathrm{S}$.

16 - Fjord, W. of the road at Röd, Förde, pos.: $59^{\circ} 35^{\prime} 20^{\prime} \mathrm{N}, 5^{\circ} 25^{\prime} 30^{\prime \prime} \mathrm{E}, 2$-VII-1971, temp. $22.4^{\circ}$

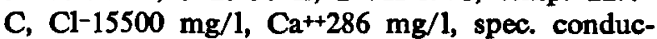
tivity $29830 \mu \mathrm{S}$.

Prov. Sogn og Fjordane:

17 - Mouth brook at N.W. point of Vetlefjorden, Svaeren, pos.: $61^{\circ} 17^{\prime} \mathrm{N}, 6^{\circ} 31^{\prime} \mathrm{E}, 6-\mathrm{VII}-1971$, temp. $10.4^{\circ} \mathrm{C}, \mathrm{Cl}-8 \mathrm{mg} / 1, \mathrm{Ca}^{++} 15 \mathrm{mg} / \mathrm{l}$, spec. conductivity $72 \mu \mathrm{S}$.

18 - Vetlefjorden, Svaeren, pos.: $61^{\circ} 17^{\prime} \mathrm{N}, 6^{\circ} 31^{\prime} \mathrm{E}$, 6-VII-1971, temp. $17.0^{\circ} \mathrm{C}, \mathrm{Cl}-18 \mathrm{mg} / 1, \mathrm{Ca}^{++20}$ $\mathrm{mg} / 1$, spec. conductivity $85 \mu \mathrm{S}$.

19 - Rockpool at Utfjorden, Anda, pos.: $61^{\circ} 52^{\prime} \mathrm{N}$, $6^{\circ} 0.5^{\prime} 30^{\prime} \mathrm{E}, 6-\mathrm{VII}-1971$, temp. $15.4^{\circ} \mathrm{C}, \mathrm{Cl}-2250$ $\mathrm{mg} / 1, \mathrm{Ca}+61 \mathrm{mg} / \mathrm{l}$, spec. conductivity $6210 \mu \mathrm{S}$.

20 - Utfjorden/Gloppenfjorden, Anda, pos.: $61^{\circ} 50^{\prime} \mathrm{N}$, $6^{\circ} 05^{\prime} 30^{\prime \prime} \mathrm{E}$, 6-VII-1971, temp. $15.2^{\circ} \mathrm{C}, \mathrm{Cl}-2000$ $\mathrm{mg} / \mathrm{l}, \mathrm{Ca}^{++48} \mathrm{mg} / \mathrm{l}$, spec. conductivity $5850 \mu \mathrm{S}$.

21 - Lusterfjord near Skjolden, pos.: $61^{\circ} 29^{\prime} 20^{\prime \prime} \mathrm{N}$, $7^{\circ} 36^{\prime} \mathrm{E}$, 3-VI- 1970 , temp. $11.2^{\circ} \mathrm{C}, \mathrm{Cl}^{-} 4440 \mathrm{mg} / 1$.

22 - Gaupnefjord near the mouth of the Jostedalselva, pos.: $61^{\circ} 24^{\prime} \mathrm{N}, 7^{\circ} 17^{\prime} 20^{\prime \prime} \mathrm{E}$, 4-VI-1970, temp. $15.3^{\circ} \mathrm{C}, \mathrm{Cl}-580 \mathrm{mg} / \mathrm{l}$.

23 - Vetlefjorden near Svearen, pos.: $61^{\circ} 17^{\prime} \mathrm{N}, 6^{\circ} 32^{\prime}$ E, 6-VI-1970, temp. $8.8^{\circ} \mathrm{C}, \mathrm{Cl}-230 \mathrm{mg} / 1$.

24 - Innvikfjorden, $10 \mathrm{~km}$ from Olden, pos.: $61^{\circ} 51^{\prime}$ $30^{\prime \prime} \mathrm{N}, 6^{\circ} 48^{\prime} \mathrm{E}, 7-\mathrm{VI}-1970$, temp. $11.4^{\circ} \mathrm{C}, \mathrm{Cl}-130$ $\mathrm{mg} / \mathrm{l}, \mathrm{Ca}^{++}<5 \mathrm{mg} / \mathrm{l}$.

25 - Sognefjord at Hella, pos.: $61^{\circ} 13^{\prime} \mathrm{N}, 6^{\circ} 36^{\prime} \mathrm{E}, 6$-VI1970 , temp. $19.0^{\circ} \mathrm{C}, \mathrm{Cl}-7800 \mathrm{mg} / \mathrm{l}, \mathrm{Ca}^{++134} \mathrm{mg} / \mathrm{l}$.

Prov. Möre og Romsdal:

26 - Killspollen, Straumshamn, pos.: $62^{\circ} 04^{\prime} \mathrm{N}, 6^{\circ} 03^{\prime}$ E, 6-VII-1971, temp. $14.1^{\circ} \mathrm{C}, \mathrm{Cl}^{-6800} \mathrm{mg} / 1, \mathrm{Ca}^{++}$ $133 \mathrm{mg} / \mathrm{l}$, spec. conductivity $17280 \mu \mathrm{S}$.

27 - Rivermouth E. of South-point Killspollen, Straumshamn, pos.: $62^{\circ} 03^{\prime} \mathrm{N}, 6^{\circ} 03^{\prime} \mathrm{E}, 6$-VII-1971, temp. $13.2^{\circ} \mathrm{C}, \mathrm{Cl}^{-5} \mathrm{mg} / \mathrm{l}, \mathrm{Ca}^{++}<5 \mathrm{mg} / \mathrm{l}$, spec. conductivity $45 \mu \mathrm{S}$.

$28-10 \mathrm{~m}$ E. of mouth river, Killspollen, Straumshamn, pos.: $62^{\circ} 03^{\prime} \mathrm{N}, 6^{\circ} 03^{\prime} \mathrm{E}$, 6-VII-1971, temp. $13.4^{\circ} \mathrm{C}, \mathrm{Cl}-2800 \mathrm{mg} / \mathrm{l}, \mathrm{Ca}+59 \mathrm{mg} / \mathrm{l}$, spec. conductivity $8010 \mu \mathrm{S}$.

29 - Rockpool at Storfjorden, Solevåg, pos.: $62^{\circ} 25^{\prime} \mathrm{N}$, $6^{\circ} 20^{\prime} \mathrm{E}, 7$-VII- 1971 , temp. $16.2^{\circ} \mathrm{C}, \mathrm{Cl}-65 \mathrm{mg} / 1$, $\mathrm{Ca}^{++}<5 \mathrm{mg} / \mathrm{l}$, spec. conductivity $270 \mu \mathrm{S}$.

30 - River running into Storfjorden, Tyssa, pos.: $62^{\circ}$ $29^{\prime} 30^{\prime \prime} \mathrm{N}, 6^{\circ} 44^{\prime} \mathrm{E}, 7-\mathrm{VII}-1971$, temp. $15.7^{\circ} \mathrm{C}, \mathrm{Cl}^{-}$ $58 \mathrm{mg} / 1, \mathrm{Ca}^{++17} \mathrm{mg} / \mathrm{l}$, spec. conductivity $240 \mu \mathrm{S}$.

31 - Storfjorden, Solevåg, pos.: $62^{\circ} 25^{\prime} \mathrm{N}, 6^{\circ} 20^{\prime} \mathrm{E}, 7$ VII-1971, temp. $14.4^{\circ} \mathrm{C}, \mathrm{Cl}^{-3} 3490 \mathrm{mg} / \mathrm{l}, \mathrm{Ca}^{++322}$ $\mathrm{mg} / \mathrm{l}$, spec. conductivity $30800 \mu \mathrm{S}$.

32 - Mouth of river W. of Isfjorden near Brevik, pos.: $62^{\circ} 35^{\prime} \mathrm{N}, 7^{\circ} 45^{\prime} \mathrm{E}, 8$-VII-1971, temp. $17.3^{\circ} \mathrm{C}$, Cl-400 mg/1, Ca ${ }^{++20} \mathrm{mg} / 1$, spec. conductivity $1260 \mu \mathrm{S}$.

33 - Isfjorden near Tokle, Andalsnes, pos.: $62^{\circ} 35^{\prime} \mathrm{N}$, $7^{\circ} 45^{\prime} \mathrm{E}, 8-\mathrm{VII}-1971$, temp. $18.2^{\circ} \mathrm{C}, \mathrm{Cl}-14800 \mathrm{mg} / 1$.

34 - Rockpool at Langfjorden, Sölsnes, pos.: $62^{\circ} 40^{\prime}$ $15^{\prime \prime} \mathrm{N}, 7^{\circ} 27^{\prime} 40^{\prime \prime} \mathrm{E}, 8-\mathrm{VII}-1971$, temp. $28.6^{\circ} \mathrm{C}, \mathrm{Cl}^{-}$ $15 \mathrm{mg} / \mathrm{l}, \mathrm{Ca}+17 \mathrm{mg} / \mathrm{l}$, spec. conductivity $170 \mu \mathrm{S}$.

35 - Pseudo-supralittoral rockpool at Kristiansund, pos.: $63^{\circ} 07^{\prime} 30^{\prime \prime} \mathrm{N}, 7^{\circ} 43^{\prime} \mathrm{E}, 8$-VIII-1971, temp. $17.4^{\circ}$ C, $\mathrm{Cl}-34 \mathrm{mg} / \mathrm{l}, \mathrm{Ca}^{++22} \mathrm{mg} / \mathrm{l}$, spec. conductivity $240 \mu$ S.

36 - Rockpool at Kristiansund, pos.: $63^{\circ} 07^{\prime} 30^{\prime \prime} \mathrm{N}$, $7^{\circ} 43^{\prime} \mathrm{E}, 8-\mathrm{VII}-1971$, temp. $18.0^{\circ} \mathrm{C}, \mathrm{Cl}-23 \mathrm{mg} / \mathrm{l}$, $\mathrm{Ca}^{++}<5 \mathrm{mg} / \mathrm{l}$, spec. conductivity $117 \mu \mathrm{S}$.

37 - Freifjorden, Kvitnes, pos.: $63^{\circ} 01^{\prime} \mathrm{N}, 7^{\circ} 47^{\prime} \mathrm{E}, 8$ -

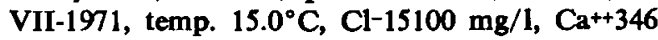
$\mathrm{mg} / \mathrm{l}$, spec. conductivity $30600 \mu \mathrm{S}$.

38 - Rockpool at Freifjorden, Kvitnes, pos.: $63^{\circ} 01^{\prime} \mathrm{N}$, $7^{\circ} 47^{\prime} \mathrm{E}, 8-\mathrm{VII}-1971$, temp. $23.0^{\circ} \mathrm{C}, \mathrm{Cl}-10500 \mathrm{mg} / \mathrm{l}$, spec. conductivity $22500 \mu \mathrm{S}$.

39 - Norddalsfjord at Eisdal, pos.: $62^{\circ} 16^{\prime} \mathrm{N}, 7^{\circ} 11^{\prime} \mathrm{E}$, 9-VI-1970, temp. $12.0^{\circ} \mathrm{C}, \mathrm{Cl}-5600 \mathrm{mg} / \mathrm{l}, \mathrm{Ca}^{++75}$ mg'l. 
40 - Rödvenfjord at Rödven, pos.: $62^{\circ} 38^{\prime} 20^{\prime \prime} \mathrm{N}, 7^{\circ} 30^{\prime}$ E, 9-VI-1970, temp. $19.4^{\circ} \mathrm{C}, \mathrm{Cl}-7000 \mathrm{mg} / \mathrm{l}, \mathrm{Ca}^{++}$ $111 \mathrm{mg} / 1$.

41 - Tingvollfjorden at Eidsöra, pos.: $62^{\circ} 48^{\prime} 30^{\prime} \mathrm{N}$, $8^{\circ} 11^{\prime} 30^{\prime \prime} \mathrm{E}, 10-\mathrm{VI}-1970$, temp. $14.0^{\circ} \mathrm{C}, \mathrm{Cl}-2700$ $\mathrm{mg} / 1, \mathrm{Ca}^{++38} \mathrm{mg} / \mathrm{l}$.

42 - Fjord at Skjölberg, island Smöla, pos.: $63^{\circ} 20^{\prime} 30^{\prime \prime}$ $\mathrm{N}, 8^{\circ} 02^{\prime} \mathrm{E}, 9$-VII-1971, temp. $18.2^{\circ} \mathrm{C}, \mathrm{Cl}-30 \mathrm{mg} / 1$,

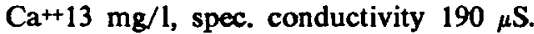

43 - Mouth brnok near Skjölberg, pos.: $63^{\circ} 20^{\prime} 30^{\prime \prime} \mathrm{N}$, $8^{\circ} 02^{\prime} \mathrm{E}$, 9-VII-1971, temp. $18.4^{\circ} \mathrm{C}, \mathrm{Cl}-4 \mathrm{mg} / 1$, $\mathrm{Ca}^{++13} \mathrm{mg} / \mathrm{l}$, spec. conductivity $160 \mu \mathrm{S}$.

44 - Geirangerfjord at Geiranger, pos.: $62^{\circ} 06^{\prime} 10^{\prime} \mathrm{N}$, $7^{\circ} 12^{\prime} 30^{\prime} \mathrm{E}, 8-\mathrm{VI}-1970$, temp. $10.2^{\circ} \mathrm{C}, \mathrm{Cl}-2430 \mathrm{mg} /$

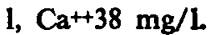

45 - Mouth and outer marches river near Tyssa, pos.: $62^{\circ} 29^{\prime} 30^{\prime \prime} \mathrm{N}, 6^{\circ} 44^{\prime} \mathrm{E}$, 7-VII-1971, temp. $15.7^{\circ} \mathrm{C}$ Cl-750 mg/1, Ca++22 mg/1, spec. conductivity $2475 \mu \mathrm{S}$.

46 - River running into Batnfjorden, $1 \mathrm{~km} \mathrm{W.} \mathrm{of}$ Batnfjordsöra, pos.: $62^{\circ} 55^{\prime} \mathrm{N}, 7^{\circ} 40^{\prime} 30^{\prime} \mathrm{E}$, 8-VII1971 , temp. $17.8^{\circ} \mathrm{C}, \mathrm{Cl}-31 \mathrm{mg} / 1, \mathrm{Ca}^{++15} \mathrm{mg} / \mathrm{l}$, spec. conductivity $160 \mu \mathrm{S}$.

Prov. Sör-Trondelag:

47 - Fröyhavet Fjord, Tuvnes, pos.: $63^{\circ} 44^{\prime} 30^{\prime \prime} \mathrm{N}, 8^{\circ} 39^{\prime}$ E, 10 -VII-1971, temp. $14.8^{\circ} \mathrm{C}, \mathrm{Cl}-39 \mathrm{mg} / 1$, spec. conductivity $220 \mu \mathrm{S}$.

48 - Storvatnet, Musdal, pos.: $63^{\circ} 35^{\prime} 30^{\prime \prime} \mathrm{N}, 9^{\circ} 38^{\prime} 5^{\prime \prime} \mathrm{E}$, 11-VII-1971, temp. $14.0^{\circ} \mathrm{C}, \mathrm{Cl}-70 \mathrm{mg} / 1, \mathrm{Ca}^{++47}$ $\mathrm{mg} / \mathrm{l}$, spec. conductivity $1190 \mu \mathrm{S}$.

49 - Rockpool at Stjörnfjorden, Mölnbukt, pos.: $63^{\circ}$ $37^{\prime} 30^{\prime \prime} \mathrm{N}, 9^{\circ} 39^{\prime} \mathrm{E}, 11-\mathrm{VII}-1971$, temp. $14.0^{\circ} \mathrm{C}, \mathrm{Cl}^{-}$ $5000 \mathrm{mg} / \mathrm{l}, \mathrm{Ca}^{++109} \mathrm{mg} / \mathrm{l}$, spec. conductivity $11340 \mu \mathrm{S}$.

50 - Stjörnfjorden at Mölnbukt, pos.: $63^{\circ} 37^{\prime} 30^{\prime} \mathrm{N}$, $9^{\circ} 39^{\prime} \mathrm{E}, 11-\mathrm{VII}-1971$, temp. $11.0^{\circ} \mathrm{C}, \mathrm{Cl}-17000 \mathrm{mg} /$ $1, \mathrm{Ca}^{++357} \mathrm{mg} / 1$, spec. conductivity $33520 \mu \mathrm{S}$.

51 - Hemnefjorden near ferry, Sunde, pos.: $63^{\circ} 29^{\prime} 30^{\prime \prime}$ $\mathrm{N}, 9^{\circ} 10^{\prime} \mathrm{E}$, 10 -VII-1971, temp. $12.0^{\circ} \mathrm{C}, \mathrm{Cl}-17000$

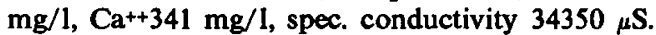

52 - Rivulet near Hemnefjorden, Sunde, pos. $63^{\circ} 29^{\prime}$ $30^{\prime \prime} \mathrm{N}, 9^{\circ} 10^{\prime} \mathrm{E}, 10-\mathrm{VII}-1971$, temp. $16.4^{\circ} \mathrm{C}, \mathrm{Cl}-20$ $\mathrm{mg} / 1, \mathrm{Ca}^{++}<5 \mathrm{mg} / \mathrm{l}$, spec. conductivity $100 \mu \mathrm{S}$.

53 - Rivermouth near ferry at Hemnefjorden, Sunde, pos.: $63^{\circ} 29^{\prime} 30^{\prime \prime} \mathrm{N}, 9^{\circ} 10^{\prime} \mathrm{E}, 10-\mathrm{VII}-1971$, temp.

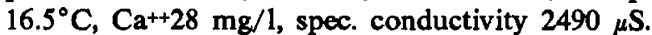

Prov. Nordland:

54 - Kolbotn, Arsandöy, pos.: $65^{\circ} 04^{\prime} \mathrm{N}, 12^{\circ} 11^{\prime} \mathrm{E}, 13$ VII-1971, temp. $10.4^{\circ} \mathrm{C}, \mathrm{Cl}-17900 \mathrm{mg} / 1, \mathrm{Ca}^{++} 296$ $\mathrm{mg} / \mathrm{l}$, spec. conductivity $27250 \mu \mathrm{S}$.

55 - Pseudo-supralittoral rockpool at Sörfjorden, Möllebogen, pos.: $65^{\circ} 03^{\prime} 30^{\prime \prime} \mathrm{N}, 12^{\circ} 04^{\prime} \mathrm{E}, 13-\mathrm{VII}-1971$, temp. $12.0^{\circ} \mathrm{C}, \mathrm{Cl}-9200 \mathrm{mg} / \mathrm{l}, \mathrm{Ca}^{++} 137 \mathrm{mg} / \mathrm{l}$, spec. conductivity $18900 \mu \mathrm{S}$.

56 - Rockpool at Holm, pos.: $65^{\circ} 10^{\prime} 30^{\prime \prime} \mathrm{N}, 12^{\circ} 07^{\prime} \mathrm{E}$, 13-VII-1971, temp. $12.0^{\circ} \mathrm{C}, \mathrm{Cl}-218 \mathrm{mg} / 1, \mathrm{Ca}++8$ $\mathrm{mg} / \mathrm{l}$, spec. conductivity $730 \mu \mathrm{S}$.

57 - Creek running into Torgfjorden, Berg, pos.: $65^{\circ}$ $23^{\prime} \mathrm{N}, 12^{\circ} 16^{\prime} 30^{\prime \prime} \mathrm{E}, 13-\mathrm{VII}-1971$, temp. $9.8^{\circ} \mathrm{C}, \mathrm{Cl}^{-}$

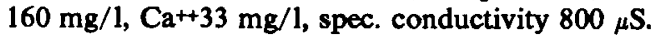

58 - Torgfjorden, Berg, pos.: $65^{\circ} 23^{\prime} \mathrm{N}, 12^{\circ} 16^{\prime} 30^{\prime \prime} \mathrm{E}$, 13-VII-1971, temp. $10.8^{\circ} \mathrm{C}, \mathrm{Cl}-15700 \mathrm{mg} / \mathrm{l}, \mathrm{Ca}^{++}$ $361 \mathrm{mg} / 1$, spec. conductivity $30740 \mu \mathrm{S}$.

59 - Rockpool at Harmfjorden, near camping Brönnöysund, pos.: $65^{\circ} 26^{\prime} 30^{\prime \prime} \mathrm{N}, 12^{\circ} 12^{\prime} \mathrm{E}, 14-\mathrm{VII}-1971$, temp. $13.2^{\circ} \mathrm{C}, \mathrm{Cl}-16100 \mathrm{mg} / 1, \mathrm{Ca}^{++276} \mathrm{mg} / \mathrm{l}$, spec. conductivity $36040 \mu \mathrm{S}$.

60 - Rockpool at Fjord at Leinesodden, pos.: $66^{\circ} 02^{\prime}$ $\mathrm{N}, 12^{\circ} 41^{\prime} \mathrm{E}, 14-\mathrm{VII}-1971$, temp. $18.5^{\circ} \mathrm{C}, \mathrm{Cl}-3800$ $\mathrm{mg} / \mathrm{l}, \mathrm{Ca}+80 \mathrm{mg} / 1$, spec. conductivity $8790 \mu \mathrm{S}$.

61 - Mouth Saksenkivelka at Saltdalsfjorden, Saltdal, pos.: $67^{\circ} 06^{\prime} 30^{\prime \prime} \mathrm{N}, 1^{\circ} 27^{\prime} \mathrm{E}, 15-\mathrm{VII}-1971$, temp. $9.5^{\circ} \mathrm{C}, \mathrm{Cl}-66 \mathrm{mg} / \mathrm{l}, \mathrm{Ca}^{++}<5 \mathrm{mg} / \mathrm{l}$, spec. conductivity $250 \mu \mathrm{S}$.

62 - Rockpool at Nedrevatnet, Fauske, pos.: $67^{\circ} 15^{\prime} \mathrm{N}$, $15^{\circ} 26^{\prime} 30^{\prime \prime} \mathrm{E}, 15-\mathrm{VII}-1971$, temp. $13.4^{\circ} \mathrm{C}, \mathrm{Cl}-1150$ $\mathrm{mg} / 1, \mathrm{Ca}^{++27} \mathrm{mg} / \mathrm{l}$, spec. conductivity $3960 \mu \mathrm{S}$.

63 - Saltdalsfjorden at Saltdal, pos.: $67^{\circ} 06^{\prime} 30^{\prime \prime} \mathrm{N}, 15^{\circ}$ $27^{\prime} \mathrm{E}, 15-\mathrm{VII}-1971$, temp. $12.6^{\circ} \mathrm{C}, \mathrm{Cl}-840 \mathrm{mg} / \mathrm{l}$, $\mathrm{Ca}^{++} 14 \mathrm{mg} / \mathrm{l}$, spec. conductivity $2550 \mu \mathrm{S}$.

64 - Fjord Fauske Vika, Fauske, pos,: $67^{\circ} 15^{\prime} \mathrm{N}, 15^{\circ}$ $27^{\prime} 30^{\prime \prime} \mathrm{E}, 15-\mathrm{VII}-1971$, temp. $13.4^{\circ} \mathrm{C}, \mathrm{Cl}-680 \mathrm{mg} /$ $1, \mathrm{Ca}^{++14} \mathrm{mg} / \mathrm{l}$, spec. conductivity $2170 \mu \mathrm{S}$.

65 - Törrfjorden, Vlegården, pos.: $67^{\circ} 22^{\prime} \mathrm{N}, 15^{\circ} 37^{\prime} \mathrm{E}$, 15-VII-1971, temp. $12.4^{\circ} \mathrm{C}, \mathrm{Cl}-51 \mathrm{mg} / 1, \mathrm{Ca}^{++}<5$ $\mathrm{mg} / \mathrm{l}$, spec. conductivity $200 \mu \mathrm{S}$.

66 - Leirfjorden, Sommarset, pos.: $67^{\circ} 32^{\prime} \mathrm{N}, 15^{\circ} 36^{\prime} \mathrm{E}$, 15-VII-1971, temp. $13.3^{\circ} \mathrm{C}, \mathrm{Cl}-3800 \mathrm{mg} / \mathrm{l}, \mathrm{Ca}^{++}$ $54 \mathrm{mg} / \mathrm{l}$, spec. conductivity $10110 \mu \mathrm{S}$.

67 - Cliffbrook N. of Kalviktunnel, Sommarset, pos.: $67^{\circ} 32^{\prime} \mathrm{N}, 15^{\circ} 36^{\prime} \mathrm{E}, 15-\mathrm{VII}-1971$, temp. $9.0^{\circ} \mathrm{C}, \mathrm{Cl}^{-}$ $2 \mathrm{mg} / \mathrm{l}, \mathrm{Ca}^{++}<5 \mathrm{mg} / \mathrm{l}$, spec. conductivity $49 \mu \mathrm{S}$.

68 - Rockpool at Leirfjorden, Sommarset, pos.: $67^{\circ}$ $32^{\prime} \mathrm{N}, 15^{\circ} 32^{\prime} \mathrm{E}, 15-\mathrm{VII}-1971$, temp. $17.0^{\circ} \mathrm{C}, \mathrm{Cl}^{-}$ $4900 \mathrm{mg} / \mathrm{l}, \mathrm{Ca}^{++112} \mathrm{mg} / \mathrm{l}$, spec. conductivity $12660 \mu \mathrm{S}$.

69 - Sagfjorden, Tömmerneset, pos.: $67^{\circ} 55^{\prime} 30^{\prime \prime} \mathrm{N}, 15^{\circ}$ 53'E, 15-VII-1971, temp. $14.2^{\circ} \mathrm{C}, \mathrm{Cl}-194 \mathrm{mg} / \mathrm{l}$, $\mathrm{Ca}^{++9} \mathrm{mg} / \mathrm{l}$, spec. conductivity $660 \mu \mathrm{S}$.

70 - Rockpool at Presteidfjorden, Hammaröy, pos.: $68^{\circ} 05^{\prime} 30^{\prime \prime} \mathrm{N}, 15^{\circ} 37^{\prime} 30^{\prime} \mathrm{E}, 15-\mathrm{VII}-1971$, temp. $13.9^{\circ}$ $\mathrm{C}, \mathrm{Cl}-2150 \mathrm{mg} / 1, \mathrm{Ca}^{++61} \mathrm{mg} / \mathrm{l}$, spec. conductivity $5850 \mu \mathrm{S}$.

71 - Rivulet on temporary dry marsh, Presteidfjorden, Hammaröy, pos.: $68^{\circ} 05^{\prime} 30^{\prime \prime} \mathrm{N}, 15^{\circ} 37^{\prime} 30^{\prime} \mathrm{E}, 15-$ VII-1971, temp. $10.4^{\circ} \mathrm{C}, \mathrm{Cl}-2450 \mathrm{mg} / 1, \mathrm{Ca}^{++63}$ $\mathrm{mg} / 1$, spec. conductivity $7200 \mu \mathrm{S}$.

72 - Rockpool at Rombaken, Fossetua, pos.: $68^{\circ} 27^{\prime}$ $\mathrm{N}, 17^{\circ} 31^{\prime} 30^{\prime \prime} \mathrm{E}, 16-\mathrm{VII}-1971$, temp. $18.4^{\circ} \mathrm{C}, \mathrm{Cl}^{-}$ $7200 \mathrm{mg} / 1, \mathrm{Ca}+143 \mathrm{mg} / 1$, spec. conductivity $17300 \mu \mathrm{S}$.

73 - Cliffstream running into Rombaken, Fossetua, pos.: $68^{\circ} 27^{\prime} \mathrm{N}, 1^{\circ} 31^{\prime} 30^{\prime \prime} \mathrm{E}, 16-\mathrm{VII}-1971$, temp. $8.2^{\circ} \mathrm{C}, \mathrm{Cl}^{-4} \mathrm{mg} / 1, \mathrm{Ca}^{++}<5 \mathrm{mg} / 1$, spec. conductivity $76 \mu \mathrm{S}$.

74 - Rombakenfjorden, Fossetua, pos.: $68^{\circ} 27^{\prime} \mathrm{N}, 17^{\circ}$ 31'30"E, 16-VII-1971, temp. $9.1^{\circ} \mathrm{C}, \mathrm{Cl}-630 \mathrm{mg} / \mathrm{l}$,

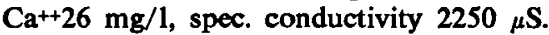

75 - Fjord near Reine, Lofoten, pos.: $67^{\circ} 56^{\prime} \mathrm{N}, 13^{\circ}$ $16^{\prime} 20^{\prime \prime} \mathrm{E}, 18-\mathrm{VIII}-1939$ (no further details known).

Prov. Troms:

76 - Gratangsbotn at Rivermouth, Fjordbotn, pos.: $68^{\circ} 40^{\prime} \mathrm{N}, 17^{\circ} 24^{\prime} \mathrm{E}, 16-\mathrm{VII}-1971$, temp. $12.0^{\circ} \mathrm{C}$, Cl-12 mg/1, Ca++13 mg/l, spec. conductivity 112 $\mu \mathrm{S}$.

77 - Sagfjorden, Ottera, pos.: $68^{\circ} 51^{\prime} 30^{\prime \prime} \mathrm{N}, 17^{\circ} 48^{\prime} \mathrm{E}$, 17-VII-1971, temp. $10.4^{\circ} \mathrm{C}, \mathrm{Cl}-2600 \mathrm{mg} / \mathrm{l}, \mathrm{Ca}^{++83}$ $\mathrm{mg} / \mathrm{l}$, spec. conductivity $6930 \mu \mathrm{S}$.

78 - Rockpool at Sagfjorden, Ottera, pos.: $68^{\circ} 51^{\prime} 30^{\prime \prime}$ $\mathrm{N}, 17^{\circ} 48^{\prime} \mathrm{E}, 17-\mathrm{VII}-1971$, temp. $12.4^{\circ} \mathrm{C}, \mathrm{Cl}-1290$ 
$\mathrm{mg} / 1, \mathrm{Ca}+48 \mathrm{mg} / 1$, spec. conductivity $3780 \mu \mathrm{S}$.

79 - Rivulet running into Balsfjorden, Kvitberg, pos.: $69^{\circ} 15^{\prime} 30^{\prime \prime} \mathrm{N}, 19^{\circ} 27^{\prime} \mathrm{E}, 17-\mathrm{VII}-1971$, temp. $9.7^{\circ} \mathrm{C}$, Cl-6 mg/1, Ca++ $<5 \mathrm{mg} / 1$, spec. conductivity 73 $\mu \mathrm{S}$.

80 - Balsfjorden, Kvitberg, pos.: $69^{\circ} 15^{\prime} 30^{\prime \prime} \mathrm{N}, 19^{\circ} 27^{\prime}$ E, $17-$ VII-1971, temp. $11.4^{\circ} \mathrm{C}, \mathrm{Cl}-13500 \mathrm{mg} / 1$,

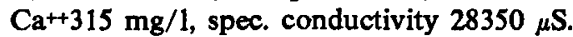

81 - Rockpool at Balsfjorden, Laksvatn, pos.: $69^{\circ} 22^{\prime}$ $30^{\prime \prime} \mathrm{N}, 19^{\circ} 21^{\prime} \mathrm{E}, 17-\mathrm{VII}-1971$, temp. $13.7^{\circ} \mathrm{C}, \mathrm{Cl}^{-}$ $4600 \mathrm{mg} / \mathrm{l}, \mathrm{Ca}+104 \mathrm{mg} / \mathrm{l}$, spec. conductivity $12150 \mu \mathrm{S}$.

82 - River from lake to Balsfjorden, Ytre Laksvatn, pos.: $69^{\circ} 23^{\prime} \mathrm{N}, 19^{\circ} 22^{\prime} \mathrm{E}, 17-\mathrm{VII}-1971$, temp. $10.4^{\circ}$ C, $\mathrm{Cl}^{-9} \mathrm{mg} / 1, \mathrm{Ca}^{++}<5 \mathrm{mg} / 1$, spec. conductivity $64 \mu \mathrm{S}$.

83 - Rockpool at Tromsöysundet, camping Tromsö, pos.: $69^{\circ} 37^{\prime} 30^{\prime \prime} \mathrm{N}, 1^{\circ} 54^{\prime} \mathrm{E}$, 17-VII-1971, temp. $13.6^{\circ} \mathrm{C}, \mathrm{Cl}-13900 \mathrm{mg} / 1, \mathrm{Ca}^{++304} \mathrm{mg} / \mathrm{l}$, spec. conductivity $31050 \mu \mathrm{S}$.

84 - Tromsöysundet, camping Tromsö, pos.: $69^{\circ} 37^{\prime} 30^{\prime \prime}$ $\mathrm{N}, 18^{\circ} 54^{\prime} \mathrm{E}, 17-\mathrm{VII}-1971$, temp. $10.4^{\circ} \mathrm{C}, \mathrm{Cl}-17500$ $\mathrm{mg} / 1, \mathrm{Ca}^{++} 367 \mathrm{mg} / \mathrm{l}$, spec. conductivity $33300 \mu \mathrm{S}$.

85 - Cliffstream running into Lyngen, Rasteby, pos.: $69^{\circ} 24^{\prime} \mathrm{N}, 20^{\circ} 09^{\prime} \mathrm{E}, 17-\mathrm{VII}-1971$, temp. $8.5^{\circ} \mathrm{C}, \mathrm{Cl}$ $3 \mathrm{mg} / 1, \mathrm{Ca}^{++}<5 \mathrm{mg} / 1$, spec. conductivity $96 \mu \mathrm{S}$.

86 - Jaegervatnet $500 \mathrm{~m}$ from the fjord, pos.: $69^{\circ} 44^{\prime}$ $\mathrm{N}, 19^{\circ} 49^{\prime} \mathrm{E}$, 19-VII-1971, temp. $10.0^{\circ} \mathrm{C}, \mathrm{Cl}-6 \mathrm{mg} /$ 1, spec. conductivity $53 \mu \mathrm{S}$.

87 - Mouth Jaegervatnet, pos.: $69^{\circ} 44^{\prime} \mathrm{N}, 19^{\circ} 49^{\prime} \mathrm{E}, 19$ VII-1971, temp. $10.0^{\circ} \mathrm{C}, \mathrm{Cl}-26 \mathrm{mg} / 1, \mathrm{Ca}^{++}<5 \mathrm{mg} /$ 1, spec. conductivity $77 \mu \mathrm{S}$.

88 - Cliffstream at Lyngen, Lyngmoen, pos.: $69^{\circ} 40^{\prime} \mathrm{N}$, $20^{\circ} 29^{\prime} 30^{\prime \prime} \mathrm{E}, 19-\mathrm{VII}-1971$, temp. $7.4^{\circ} \mathrm{C}, \mathrm{Cl}-10 \mathrm{mg} /$ $1, \mathrm{Ca}^{++}<5 \mathrm{mg} / \mathrm{l}$, spec. conductivity $68 \mu \mathrm{S}$.

89 - Rockpool at Straumfjorden, pos.: $69^{\circ} 51^{\prime} \mathrm{N}, 21^{\circ}$ $13^{\prime} \mathrm{E}, 19-\mathrm{VII}-1971$, temp. $13.3^{\circ} \mathrm{C}, \mathrm{Cl}-11400 \mathrm{mg} / \mathrm{l}$,

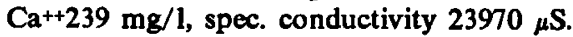

90 - Cliffstream running into Straumfjorden, Straumfjordnes, pos.: $69^{\circ} 51^{\prime} \mathrm{N}, 21^{\circ} 13^{\prime} \mathrm{E}, 19-\mathrm{VII}-1971$, temp. $10.2^{\circ} \mathrm{C}, \mathrm{Cl}-4 \mathrm{mg} / 1, \mathrm{Ca}^{++}<5 \mathrm{mg} / 1$, spec. conductivity $79 \mu \mathrm{S}$.

91 - Straumfjorden at Straumfjordnes, pos.: $69^{\circ} 51^{\prime} \mathrm{N}$, $21^{\circ} 13^{\prime} \mathrm{E}, 19-\mathrm{VII}-1971$, temp. $12.4^{\circ} \mathrm{C}, \mathrm{Cl}-10900$

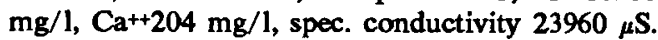

92 - Mouth Navetkjokka in Straumen, Navet, pos.: $69^{\circ} 47^{\prime} \mathrm{N}, 21^{\circ} 56^{\prime} \mathrm{E}, 19-\mathrm{VII}-1971$, temp. $8.3^{\circ} \mathrm{C}$, spec. conductivity $230 \mu \mathrm{S}$.

93 - Rockpool at Straumen, Kjackan, pos.: $69^{\circ} 46^{\prime} 30^{\prime \prime} \mathrm{N}$, $22^{\circ} 05^{\prime} \mathrm{E}, 19-\mathrm{VII}-1971$, temp. $14.1^{\circ} \mathrm{C}, \mathrm{Cl}-11600$

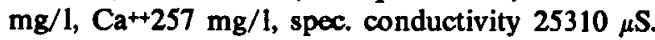

94 - Little stream on tidal marsh S. of Alteidelva, Alteidet, pos.: $70^{\circ} 01^{\prime} 30^{\prime \prime} \mathrm{N}, 22^{\circ} 05^{\prime} \mathrm{E}, 19-\mathrm{VII}-1971$, temp. $7.4^{\circ} \mathrm{C}, \mathrm{Cl}-10 \mathrm{mg} / \mathrm{l}, \mathrm{Ca}++5 \mathrm{mg} / \mathrm{l}$, spec. conductivity $80 \mu \mathrm{S}$.

95 - Mouth river, S. of Alteidelva, Aldeidet, pos.: $70^{\circ}$ $01^{\prime} 30^{\prime} \mathrm{N}, 20^{\circ} 05^{\prime} \mathrm{E}, 19-\mathrm{VII}-1971$, temp. $8.3^{\circ} \mathrm{C}, \mathrm{Cl}-$ $12700 \mathrm{mg} / \mathrm{l}, \mathrm{Ca}^{++302} \mathrm{mg} / 1$, spec. conductivity $23970 \mu \mathrm{S}$.

96 - Mouth of the Malselva, Nordstrand, pos.: $69^{\circ} 16^{\prime}$ $30^{\prime \prime} \mathrm{N}, 18^{\circ} 30^{\prime} 30^{\prime \prime} \mathrm{E}$, $17-\mathrm{VII}-1971$, temp. $11.6^{\circ} \mathrm{C}$, $\mathrm{Cl}-27 \mathrm{mg} / 1, \mathrm{Ca}+17 \mathrm{mg} / \mathrm{l}$, spec. conductivity 170 $\mu \mathrm{S}$.

Prov. Finmark:

97 - Rockpool at Altafjorden, Inestoften, pos.: $70^{\circ} 08^{\prime}$ $30^{\prime} \mathrm{N}, 22^{\circ} 59^{\prime} 30^{\prime} \mathrm{E}, 19-\mathrm{VII}-1971$, temp. $11.0^{\circ} \mathrm{C}$,

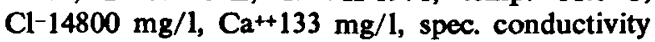
$28120 \mu \mathrm{S}$.

98 - Beach of Altafjorden, under boulders, Inestoften, pos.: $70^{\circ} 08^{\prime} 30^{\prime \prime} \mathrm{N}, 22^{\circ} 59^{\prime} 30^{\prime \prime} \mathrm{E}$, 19-VII-1971, no water sample.

99 - Altafjorden at Inestoften, pos.: $70^{\circ} 08^{\prime} 30^{\prime} \mathrm{N}, 22^{\circ}$ $59^{\prime} 30^{\prime \prime} \mathrm{E}, 19-\mathrm{VII}-1971$, temp. $10.6^{\circ} \mathrm{C}, \mathrm{Cl}-16200$ $\mathrm{mg} / 1$, spec. conductivity $28930 \mu \mathrm{S}$.

100 - Rockpool at Altafjorden, Rafsbotn, pos.: $70^{\circ} \mathrm{N}$, $23^{\circ} 29^{\prime} 30^{\prime \prime} \mathrm{E}, 21-\mathrm{VII}-1971$, temp. $19.4^{\circ} \mathrm{C}, \mathrm{Cl}-390$ $\mathrm{mg} / \mathrm{l}, \mathrm{Ca}+328 \mathrm{mg} / \mathrm{l}$, spec. conductivity $1310 \mu \mathrm{S}$.

101 - Altafjorden, Rafsbotn, pos.: $70^{\circ} \mathrm{N}, 23^{\circ} 29^{\prime} 30^{\prime \prime} \mathrm{E}$, 21-VII-1971, temp. $14.2^{\circ} \mathrm{C}$, spec. conductivity $11530 \mu \mathrm{S}$.

102 - Rockpool at Porsangen, Repvåg, pos.: $70^{\circ} 45^{\prime} \mathrm{N}$, $25^{\circ} 41^{\prime} \mathrm{E}, 21-\mathrm{VII}-1971$, temp. $10.4^{\circ} \mathrm{C}, \mathrm{Cl}-4500 \mathrm{mg} /$ 1 , spec. conductivity $640 \mu \mathrm{S}$.

103 - Rockpool at Nordkapp, pos.: $71^{\circ} 09^{\prime} 30^{\prime \prime} \mathrm{N}, 25^{\circ} 48^{\prime}$ $30^{\prime} \mathrm{E}, 22-\mathrm{VII}-1971$, temp. $9.6^{\circ} \mathrm{C}, \mathrm{Cl}-7400 \mathrm{mg} / \mathrm{l}$, $\mathrm{Ca}+96 \mathrm{mg} / \mathrm{l}$, spec. conductivity $11530 \mu \mathrm{S}$.

104 - Rockpool at Risfjorden, Skarsvåg, pos.: $71^{\circ} 06^{\prime} 30^{\prime \prime}$ $\mathrm{N}, 25^{\circ} 49^{\prime} 30^{\prime \prime} \mathrm{E}, 22-\mathrm{VII}-1971$, temp. $9.0^{\circ} \mathrm{C}, \mathrm{Cl}-$ $4600 \mathrm{mg} / \mathrm{l}, \mathrm{Ca}^{++84} \mathrm{mg} / 1$, spec. conductivity 11060 $\boldsymbol{\mu S}$.

105 - Two rockpoois at Porsangen, Nordmanset, pos.: $70^{\circ} 06^{\prime} \mathrm{N}, 26^{\circ} 16^{\prime} \mathrm{E}, 22-\mathrm{VII}-1971$, temp. $8.6^{\circ} \mathrm{C}, \mathrm{Cl}$ $20 \mathrm{mg} / 1, \mathrm{Ca}^{++}<5 \mathrm{mg} / \mathrm{l}$, spec. conductivity $110 \mu \mathrm{S}$.

106 - Cliffstream at Porsangen, Ytre Gåradak, pos.: $70^{\circ} 15^{\prime} 30^{\prime \prime} \mathrm{N}, 25^{\circ} 02^{\prime} \mathrm{E}, 22-\mathrm{VII}-1971$, temp. $10.6^{\circ} \mathrm{C}$, $\mathrm{Cl}-10600 \mathrm{mg} / 1, \mathrm{Ca}^{++} 228 \mathrm{mg} / 1$, spec. conductivity $23960 \mu \mathrm{S}$.

107 - Porsangen, Nordmanset, pos.: $70^{\circ} 06^{\prime} \mathrm{N}, 20^{\circ} 16^{\prime} \mathrm{E}$, 22-VII-1971, temp. $11.2^{\circ} \mathrm{C}, \mathrm{Cl}-16900 \mathrm{mg} / 1, \mathrm{Ca}^{++}$ $316 \mathrm{mg} / 1$, spec. conductivity $32270 \mu \mathrm{S}$.

108 - Rockpools at Austerbotn, Stråskogen, pos.: $70^{\circ}$ $04^{\prime} \mathrm{N}, 25^{\circ} 09^{\prime} \mathrm{E}, 22-\mathrm{VII}-1971$, temp. $16.0^{\circ} \mathrm{C}, \mathrm{Cl}^{-}$ $7700 \mathrm{mg} / \mathrm{l}, \mathrm{Ca}^{++} 198 \mathrm{mg} / \mathrm{l}$, spec. conductivity $18080 \mu \mathrm{S}$.

109 - Austerbotn, Stråskogen, pos.: $70^{\circ} 04^{\prime} \mathrm{N}, 25^{\circ} 09^{\prime} \mathrm{E}$, 22-VII-1971, temp. $11.5^{\circ} \mathrm{C}, \mathrm{Cl}-14100 \mathrm{mg} / \mathrm{l}, \mathrm{Ca}^{++}$ $327 \mathrm{mg} / \mathrm{l}$, spec. conductivity $31350 \mu \mathrm{S}$.

110 - Rockpool at Laksefjorden, Landersfjorden, pos.: $70^{\circ} 25^{\prime} 30^{\prime \prime} \mathrm{N}, \quad 26^{\circ} 43^{\prime} 30^{\prime \prime} \mathrm{E}, 22-\mathrm{VII}-1971$, temp. $12.0^{\circ} \mathrm{C}, \mathrm{Cl}-12100 \mathrm{mg} / \mathrm{l}, \mathrm{Ca}^{++261} \mathrm{mg} / 1$, spec. conductivity $24890 \mu \mathrm{S}$.

111 - Two rockpools at Munkfjorden, Munknes, pos.: $69^{\circ} 42^{\prime} \mathrm{N}, 29^{\circ} 37^{\prime} \mathrm{E}, 23-\mathrm{VII}-1971$, temp. $16.2^{\circ} \mathrm{C}$, $\mathrm{Cl}-10000 \mathrm{mg} / 1, \mathrm{Ca}^{++204} \mathrm{mg} / 1$, spec. conductivity $23050 \mu \mathrm{S}$.

112 - Cliffstream at Munkfjorden, Munknes, pos.: $69^{\circ}$ $42^{\prime} \mathrm{N}, 29^{\circ} 37^{\prime} \mathrm{E}, 23-\mathrm{VII}-1971$, temp. $11.2^{\circ} \mathrm{C}, \mathrm{Cl}-$ $17000 \mathrm{mg} / \mathrm{l}$.

113 - Langfjorden at Straumdalen, pos.: $69^{\circ} 42^{\prime} 30^{\prime \prime} \mathrm{N}$, $29^{\circ} 57^{\prime} \mathrm{E}, 23-\mathrm{VII}-1971$, temp. $12.4^{\circ} \mathrm{C}, \mathrm{Cl}-11700$

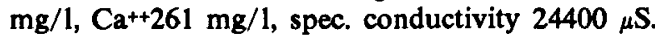

114 - Veinesbukta at Grasbakken, pos.: $70^{\circ} 05^{\prime} \mathrm{N}, 28^{\circ}$ 47'E, 23-VII-1971, temp. $9.8^{\circ} \mathrm{C}, \mathrm{Cl}-17900 \mathrm{mg} / \mathrm{l}$,

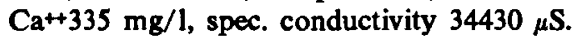

115 - Rockpool at Veinesbukta, Grasbakken, pos.: $70^{\circ}$ $05^{\prime} \mathrm{N}, 28^{\circ} 47^{\prime} \mathrm{E}, 23-\mathrm{VII}-1971$, temp. $12.8^{\circ} \mathrm{C}, \mathrm{Cl}^{-}$ $14000 \mathrm{mg} / 1, \mathrm{Ca}+341 \mathrm{mg} / 1$, spec. conductivity $32540 \mu \mathrm{S}$.

116 - Rockpool at Verangerfjorden, Saltfjern, pos.: $70^{\circ}$ $03^{\prime} 30^{\prime \prime} \mathrm{N}, 30^{\circ} \mathrm{E}, 24-\mathrm{VII}-1971$, temp. $17.0^{\circ} \mathrm{C}, \mathrm{Cl}-$ 
$14900 \mathrm{mg} / 1, \mathrm{Ca}^{++322} \mathrm{mg} / \mathrm{l}$, spec. conductivity $28930 \mu \mathrm{S}$.

117 - Rockpool at Verangerfjorden, Saltfjern, pos.: $70^{\circ}$ $03^{\prime} 30^{\prime \prime} \mathrm{N}, 30^{\circ} \mathrm{E}, 24-\mathrm{VII}-1971$, temp. $18.4^{\circ} \mathrm{C}, \mathrm{Cl}^{-}$ $17600 \mathrm{mg} / \mathrm{l}, \mathrm{Ca}++312 \mathrm{mg} / \mathrm{l}$, spec. conductivity
$35330 \mu \mathrm{S}$.

118 - Verangerfjorden at Saltfjern, pos.: $70^{\circ} 03^{\prime} 30^{\prime \prime} \mathrm{N}$, $30^{\circ} \mathrm{E}, 24-\mathrm{VII}-1971$, temp. $15.4^{\circ} \mathrm{C}, \mathrm{Cl}-17700 \mathrm{mg} / \mathrm{l}$,

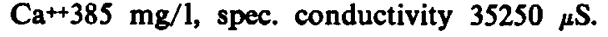

\section{REFERENCES}

Brattegard, T., 1966. The natural history of the Hardangerfjord, 7. Horizontal distribution of the fauna of rocky shores. Sarsia, $22: 1-54$.

DAHL, E., 1938. Two new Amphipoda of the genus Gammarus from Finnmark. K. norske Vidensk. Selsk. Forh., 10 (34) : 125-128.

,- 1946 . Notes on some Amphipoda from the Gullmar Fiord. Arkiv Zool., 38A (8) : 1-8.

,- 1952 . Some aspects of the ecology and the zonation of the fauna in sandy beaches. Oikos, $4: 1-27$.

Dennert, H. G., A. L. Dennert, S. Pinkster \& J. H. STOCK, 1969. Upstream and downstream migrations in relation to the reproductive cycle and to environmental factors in the amphipod Gammarus zaddachi. Bijdr. Dierk., 39 : $11-43$.

HARTOG, C. DEN, 1964. The amphipods of the Deltaic region of the rivers Rhine, Meuse and Scheldt in relation to the hydrography of the area, 3. Gammaridae. Neth. J. Sea Res., 2 (3) : 407-457.

HyNes, H. B. N., 1954. The ecology of Gammarus duebeni Lilljeborg and its occurrence in fresh water in western Britain. J. anim. Ecol., 23 : 38-84.

JONES, N. S., 1948. The ecology of the Amphipoda of the south of the Isle of Man. J. mar. biol. Ass. U.K., 27 (2) : $400-439$.

KINNE, O., 1952. Zum Lebenszyklus von Gammarus duebeni Lillj., nebst einigen Bemerkungen zur Biologie von Gammarus zaddachi Sexton subsp. zaddachi Spooner. Veröff. Inst. Meeresforsch. Bremerh., 1: 187-203.

-, 1953a. Zur Biologie und Physiologie von Gammarus duebeni Lillj. Zeitschr. wiss. Zool., 157 : 427-491.

-, 1953b. Wird die Häutungsfolge der Amphipoden (Gammarus zaddachi zaddachi Sexton, G. zaddachi salinus Spooner, G. duebeni Lillj.) durch die lunäre Periodicität beeinflusst? Kieler Meeresforsch., 9 : 271-279.

- 1954. Die Gammarus-Arten der Kieler Bucht. Zool. Jb. (Syst.), $82: 405-424$.

Meadows, P. S. \& J. I. Campbell, 1972. Habitat selection by aquatic invertebrates. Adv. mar. Biol., 10 : $271-382$.

Ofstad, K. \& J. O. Solem, 1966. Funn av Gammarus duebeni Lilljeborg i ferskvann i Trøndelag. Fauna, Oslo, 19 (3) : 149-159.

ØKLaND, J., 1959. Om tangloppen Gammarus duebeni som ble funnet ved Borrevann i sommer. Fauna, Oslo, 12 (1) : $1-14$.

ØKLAND, K. A., 1965. Om krepsdyr av slekten Gammarus i freskvann of brakkvann i Norge. Fauna, Oslo, 18 (2) : $53-62$.

,- 1969 a. On the distribution and ecology of Gammarus lacustris G. O. Sars in Norway, with notes on its morphology and biology. Nytt Mag. Zool., 17 (2) : $111-152$.
-, 1969b. List of localities with Gammarus lacustris G. O. Sars in Norway, with references and notes. Suppl. Contr. 89, Zoological Museum, University of Oslo (Mimeographed, 36 pp.).

-, 1970. Om marfloen, Gammarus duebeni, i ferskvann og et nytt funn fra Sør-Trøndelag. Fauna, Oslo, 23 : $190-195$.

Oldevig, H., 1933. Sveriges Amphipoder. Göteborgs K. Vetensk.-o. vitierhSamh. Handl., (5) (B) 3 (4) : 1282.

-, 1959. Arctic, subarctic and scandinavian amphipods in the collections of the Swedish Natural History Museum in Stockholm. Göteborgs K. Vetensk.-o. vitterh Samh. Handl., (7) (B) 8 (2) : 1-132.

Pinkster, S., A. L. Dennert, B. Stock \& J. H. Stock, 1970. The problem of european freshwater populations of Gammarus duebeni Liljeborg, 1852. Bijdr. Dierk., 40 (2) : 116-147.

RoBeluS, R. A., unpubl. De oecologische verspreiding van Amphipoda, behorende tot de familie Gammaridae, en van de Isopoda, behorende tot de familie Sphaeromidae in schokbiotopen van het supra- en eulitoraal van de Franse kanaalkust. (Doctoraalverslag, I.T.Z., Amsterdam, 1970).

SChellenberG, A., 1942. Kretstiere oder Crustacea, 4. Flohkrebse oder Amphipoda. Tierw. Dtl., 40 : 1-252.

SEgerstrále, S. G., 1947. New observations on the distribution and morphology of the amphipod, Gammarus zaddachi Sexton, with notes on related species. J. mar. biol. Ass. U.K., 27 : $219-244$.

-, 1948. On collections of Gammarus (Amphipoda) from Arctic waters (coast Petsamo-Kanin, coast of Siberia). Commentat. biol., 10 (6) : 1-13.

- , 1959. Synopsis of data on the crustaceans Gammarus locusta, Gammarus oceanicus, Pontoporeia affinis and Corophium volutator (Amphipoda Gammaridae). Commentat. biol., 20 (5) : 1-23.

SERVENTY, D. L., 1935. Observations on Gammarus zaddachi Sexton, an estuarine amphipod, and associated forms. Internat. Rev. gesamt. Hydrobiol. Hydrogr., 32 : $285-294$.

Sexton, E. W., 1912. Some brackish-water Amphipoda from the mouths of the Weser and Elbe, and from the Baltic. Proc. zoo:. Soc. Lonion, 1912 : 656-665.

,- 1942 . The relation of Gammarus zaddachi Sexton to some other species of Gammarus occurring in fresh, estuarine and marine waters. J. mar. biol. Ass. U.K., 25 : $575-606$.

Sexton, E. W. \& G. M. Spooner, 1940. An account of Marinogammarus (Schellenberg) gen. nov. (Amphipoda), with a description of a new species, $M$. pirloti. J. mar. biol. Ass. U.K., 24 : 633-682.

SkeT, B., 1971. Zur Zoologie und Phylogenie der Gammarini (Amphipoda). Bull. scient. Cons. Acad. RPF Yougosl., (A) $16: 6$. 
SOLEM, J. O., 1969. Ecology of Gammarus duebeni Lilljeborg in a Norwegian lake. K. norske Vidensk. Selsk. Skr., 1969 (2) : $1-12$.

SPOONER, G. M., 1951. On Gammarus zaddachi Segerstråle. J. mar. biol. Ass. U.K., 30 : 129-147.

Steele, V. J. \& D. H. Steele, 1972. The biology of Gammarus (Crustacea, Amphipoda) in the northwestern Atlantic, 5. Gammarus oceanicus Segerstråle. Can. J. Zool., 50 (6) : 801-813.

STEEN, E., 1951. Ecological observations on some Gammarus and Marinogammarus species on the Scandinavian west coasts. Oikos, 3 : 232-242.

STEPHENSEN, K., 1935-1942. The Amphipoda of northern Norway and Spitsbergen with adjacent waters. Troms $\phi$ Mus. Skr., 3 : 1-526.

STOCK, J. H., 1967. A revision of the European species of the Gammarus locusta-group (Crustacea, Amphipoda). Zool. Verh. Leiden, 90 : $1-56$.

STock, J. H. \& P. KANr, 1966. What is Gammarus campylops of Sars, 1894? Bull. zool. Mus. Univ. Amsterdam, 1 (2) : 7-17.

STOCK, J. H., H. NiJsSen, \& P. KANT, 1966. La répartition écologique des Amphipodes de la famille des Gammaridae dans la Slack et son estuaire. Bull. zool. Mus. Univ. Amsterdam, 1 (3) : 19-29.
SUTCLIFFE, D. W., 1967. A re-examination of the observations on the distribution of Gammarus duebeni Lilljeborg in relation to the salt content in fresh water. J. anim. Ecol., 36 : 579-597.

,- 1971 . Regulation of water and some ions in gammarids (Amphipoda), 1. Gammarus duebeni Lilljeborg from brackish water and fresh water. J. exp. Biol., 55 : $325-344$.

,- 1972 . An examination of subspecific differences in the merus of the fifth walking leg of the amphipod Gammarus duebeni Lilljeborg. Freshwat. Biol., 2 : 203216.

VADER, W., 1969. Notes on a collection of Amphipoda from the Trondheimsfjord area. $K$. norske Vidensk. Selsk. Skr., 1969 (3) : 1-20.

-, 1971. Additions to the Amphipoda of northern Norway. Astarte, $4: 47-51$.

- , 1972a. Nye funn av Gammarus zaddachi i Vest- og Nord-Norge. Fauna, Oslo, 25 : 35-38.

-, 1972b. Amfipode-slektene Gammarus og Marinogammarus i Norge, med en illustrert bestemmelsestabell til de nord-vest Europeiske arter. (Mimeographed, Biologisk stasjon, Bergen : 23 pp.).

Vlasblom, A. G. \& G. Bolier, 1971. Tolerance of embryos of Marinogammarus marinus and Orchestia gamarella (Amphipoda) to lowered salinities. Neth. J. Sea Res., 5 (3) : 334-341. 